\title{
Physical layer metrics for vertical handover toward OFDM-based networks
}

\author{
Mohamed Rabie Oularbi, Francois-Xavier Socheleau, Sebastien Houcke and Abdeldjalil Aïssa-El-Bey
}

\begin{abstract}
The emerging trend to provide users with ubiquitous seamless wireless access leads to the development of multimode terminals able to smartly switch between heterogeneous wireless networks. This switching process known as vertical handover requires the terminal to first measure various network metrics relevant to decide whether to trigger a vertical handover $(\mathrm{VHO})$ or not. This paper focuses on current and next-generation networks that rely on an OFDM physical layer with either a CSMA/CA or an OFDMA multiple-access technique. Synthesis of several signal feature estimators is presented in a unified way in order to propose a set of complementary metrics (SNR, channel occupancy rate, collision rate) relevant as inputs of vertical handover decision algorithms. All the proposed estimators are "non-data aided" and only rely on a physical layer processing so that they do not require multimode terminals to be first connected to the handover candidate networks. Results based on a detailed performance study are presented to demonstrate the efficiency of the proposed algorithms. In addition, some experimental results have been performed on a RF platform to validate one of the proposed approaches on real signals.
\end{abstract}

\section{Introduction}

Nowadays, we are facing a wide deployment of wireless networks such as 3G (LTE), WiMAX, Wifi, etc. These networks use different radio access technologies and communication protocols and belong to different administrative domains; their coexistence makes the radio environment heterogeneous.

In such environment, one possible approach to overcome the spectrum scarcity is to develop multimode terminals able to smartly switch from one wireless interface to another while maintaining IP or voice connectivity and required quality of service (QoS). This switching process is known as vertical handover or vertical handoff. This new concept will not only provide the user with a great flexibility for network access and connectivity but also generate the challenging problem of mobility support among different networks. Users will expect to continue their connections without any disruption when they move from one network to another.

The vertical handover process can be divided into three main steps $[1,2]$, namely system discovery, handoff decision, and handoff execution. During the system

\footnotetext{
* Correspondence: mohamed.oularbi@telecom-bretagne.eu Institut Télécom, Télécom Bretagne, UMR CNRS 3192 Lab-STICC Université Europenne de Bretagne, Brest, France
}

discovery step, the mobile terminals equipped with multiple interfaces have to determine which networks can be used and the services available in each network. These wireless networks may also advertise the supported data rates for different services. During the handoff decision step, the mobile device determines which network it should connect to. The decision may depend on various parameters or handoff metrics including the available bandwidth, delay, jitter, access cost, transmit power, current battery status of the mobile device, and even the user's preferences. Finally, during the handoff execution step, the connections need to be re-routed from the existing network to the new network in a seamless manner [3].

Cognitive radio appears as a highly promising solution to this combined problems. Cognitive radio systems can sense their RF environment and react, either proactively or reactively, to external stimuli [4-7]. By the term react, it is implied that the systems have the ability to reconfigure the algorithms and its communication parameters to better adapt to environment conditions. Thus, in principle, the operation of a cognitive radio system includes two stages: sense and decide [8].

This paper focuses on the sensing task. Indeed, we deal with the passive estimation of metrics that help to 
trigger a vertical handover toward OFDM -based systems such as WiFi, WiMAX, or 3G(LTE). It should be noted that the decision step and the handoff execution are not treated in this paper. These tasks may need interaction with the higher layers to guarantee a seamless and proactive vertical handover, which is beyond the scope of this paper. In the context of vertical handover, only the passive estimation is relevant since the terminal seeks to know a priori whether a network satisfies its QoS needs without wasting time and power to get connected to this network. The main contribution of this work relies on the fact that all the proposed metrics are estimated from the physical layer signal and require no connection to the system, no signal demodulation, and no frame decoding. To the best of our knowledge, various $\mathrm{VHO}$ decision algorithms based on a MAC-layer sensing have been proposed $[1,2,9-12]$, but none have been investigated on the PHY layer.

Three relevant and complementary metrics are presented. First, we propose a method to estimate the downlink signal-to-noise ratio (SNR). The SNR is an indicator commonly used to evaluate the quality of a communication link. The proposed method exploits the correlation as well as the cyclostationarity induced by the OFDM cyclic prefix (CP) to estimate the noise as well as the signal power of OFDM signals transmitted through unknown multi-path fading channel. In addition to the downlink signal quality, some knowledge on the traffic activity can be very informative since it is a good indicator of the network load. Measures of traffic activity strongly depend on the medium access technique of the sensed network. Today, OFDM wireless networks rely either on CSMA/CA (carrier sense multiple-access/ collision avoidance), see Wifi networks for instance, or on OFDMA (orthogonal frequency division multiple access), see WiMAX and 3G(LTE). Concerning the CSMA/CA protocol, we propose to estimate the channel occupancy rate (combined uplink and downlink) and the uplink collision rate, which are two relevant metrics of network load. These metrics can be estimated at the signal level providing that the terminal is equipped of several receiving antennas. For the OFDMA access techniques, the network traffic is estimated through the downlink time-frequency activity rate of the channel. Since OFDMA networks use either synchronous time division duplexing or frequency division duplexing, no collision occurs so that the collision rate metric is irrelevant $^{\mathrm{a}}$.

The rest of the paper is organized as follows: First, we deal with metrics dedicated to CSMA/CA-based networks. In Section 2.1, we present a SNR estimator dedicated to OFDM-based physical layers. Section 2.2 describes the proposed algorithms to estimate the channel occupancy rate of a CSMA/CA-based network. A first algorithm is presented in Section 2.2.3. Then, due to some limitations of the latter, in Section 2.2.5, we propose a second algorithm based on a Parzen estimator, which shown its robustness thanks to simulations. As a complementary metric, in the congested networks, we propose to estimate the channel occupancy rate. The algorithm is derived in Section 2.3, for channels with different lengths on the antennas. Section 3 deals with OFDMA-based systems. In Section 3.1, we show how the proposed SNR estimator can also be applied for OFDMA-based systems, and in Section 3.2, we describe the proposed algorithm for the estimation of the time-frequency activity rate of OFDMA signals. A proposed architecture of the receiver, based on software-defined radio is described in Section 4. All the proposed algorithms are evaluated thanks to computer simulations in Section 5. In addition, some experimental results for the channel occupancy rate are also presented in this Section 5.1.4. These results are presented for the first time; many scenarios have been driven to show how the channel occupancy rate is informative about the QoS available in a sensed networks. Furthermore, thanks to these experimentations, we are now able to say that for the case of congested networks, the channel occupancy rate itself is not sufficient enough to decide whether to trigger the handover or not and that the collision rate is a necessary complementary metric. Finally, we outline some conclusions in Section 6.

\section{Metrics for CSMA/CA based networks}

CSMA/CA is a protocol for carrier transmission in some wireless networks. Unlike CSMA/CD (carrier sense multiple-access/collision detect), which deals with transmissions after a collision has occurred, CSMA/CA acts to prevent collisions before they happen.

In CSMA/CA, as soon as a node receives a packet to be sent, it checks whether the channel is idle (no other node is transmitting at the time). If the channel is sensed "idle", then the node is permitted to begin the transmission process. If the channel is sensed as "busy", the node defers its transmission for a random period of time called backoff. If the channel is idle when the backoff counter reaches zero, the node transmits the packet. If the channel is occupied when the backoff counter reaches zero, the backoff factor is set again, and the process is repeated.

In this section, we deal with CSMA/CA networks whose physical layer is based on the OFDM modulation scheme. First, we present an algorithm for SNR estimation, then we propose a method for estimating the channel occupancy rate and finally a collision rate estimator is detailed. 


\subsection{OFDM signals SNR estimation}

SNR is an important metric that indicates the link quality. We propose a blind estimation approach, based on the correlation and the cyclostationarity induced by the OFDM CP. Assuming that an OFDM symbol consists of $\mathcal{N}_{s c}$ subcarriers, the discrete-time baseband equivalent transmitted signal is given by

$$
x(m)=\sqrt{\frac{E_{s}}{\mathcal{N}_{s c}}} \sum_{k=0}^{\mathcal{M}_{s}-1} \sum_{n=0}^{\mathcal{N}_{s c}-1} a_{k, n} e^{2 i \pi} \frac{n}{\mathcal{N}_{s c}}\left(m-D-k\left(\mathcal{N}_{x x}+D\right)\right) g\left(m-k\left(\mathcal{N}_{s c}+D\right)\right) .
$$

where $\mathcal{M}_{s}$ denotes the number of OFDM symbols in the observation window, $E_{s}$ is the average available power, and $a_{k, n}$ are the transmitted data symbols at the $n$th subcarrier of the $k$ th OFDM block. These data symbols are assumed to be independent identically distributed (i.i.d), $D$ is the cyclic prefix (CP) length, and $m \mapsto g$ $(m)$ is the pulse shaping filter.

Let $\{h(l)\}_{l}=0, \ldots, L-1$ be a baseband equivalent discretetime Rayleigh fading channel impulse response of length $L$ with $L<D$. The received samples of the OFDM signal are then expressed as

$$
y(m)=\sum_{l=0}^{L-1} h(l) x(m-l)+w(m),
$$

where $w(m)$ is an additive white Gaussian noise such that $w(m) \sim \mathcal{C N}\left(0, \sigma_{w}^{2}\right)$. The signal-to-noise ratio (SNR) is expressed as

$$
\begin{aligned}
& \operatorname{SNR}=\frac{S}{\sigma_{w}^{2}}, \\
& S=E_{s} \mathbb{E}\left[\left|a_{k, n}\right|^{2}\right] \sum_{l=0}^{L-1} \sigma_{h(l)}^{2} .
\end{aligned}
$$

where $\mathbb{E}[$.$] stands for the expectation operator. To get$ the SNR, first we have to estimate the noise power $\sigma_{w}^{2}$, and then, the power of the received signal $S$.

\subsubsection{Noise power estimation}

To estimate the noise variance, we propose to take advantage of OFDM signals' structure. More precisely, redundancy was induced by the $\mathrm{CP}$; in fact, the $\mathrm{CP}$ leads to $x\left(k\left(\mathcal{N}_{s c}+D\right)+m\right)=x\left(k\left(\mathcal{N}_{s c}+D\right)+\mathcal{N}_{s c}+m\right), \quad \forall k \in \mathbb{Z}$, and $\forall m \in\{0, \ldots, D-1\}$. Assuming a perfect synchronization and a time-invariant channel over an OFDM symbol duration, we can get $D-L$ noise variance estimates defined as

$$
\begin{aligned}
\hat{\sigma}_{w, u}^{2}= & \frac{1}{2 \mathcal{M}_{s}(D-u)} \sum_{k=0}^{\mathcal{M}_{s}-1} \sum_{m=u}^{D-1} \mid y\left(k\left(\mathcal{N}_{s c}+D\right)+m\right) \\
& -\left.\gamma\left(k\left(\mathcal{N}_{s c}+D\right)+\mathcal{N}_{s c}+m\right)\right|^{2}, \quad L \leq u \leq D-1 .
\end{aligned}
$$

The estimator with the smallest variance is found for $u=L$. The difficulty is then to estimate $L$. In [13], we proposed an estimator of $L$ inspired from maximum likelihood estimation. This estimator has the major advantage of being independent of any threshold level and shows good performance compared to the threshold-based technique proposed in [14]. Here presented method has a computational complexity (C.C) of $\mathcal{O}\left(\mathcal{M}_{s} \cdot D^{2}\right)$.

\subsubsection{Signal power estimation}

We here propose to use the cyclostationary statistics induced by the CP [15] to estimate the signal power. A signal power estimate can be given by

$$
\hat{S}=\frac{1}{2 N_{c}+1}\left|\sum_{q=-N_{c}}^{N_{c}} \hat{R}_{\gamma}^{q \alpha_{0}}(\mathcal{N}) \frac{\sin \left(\pi q \alpha_{0}\right)}{\alpha_{0} \sin \left(\pi q \alpha_{0} D\right)} e^{i \pi q \alpha_{0}(D-1)}\right|,
$$

where $\alpha_{0}=1 /\left(\mathcal{N}_{s c}+D\right)$ and

$$
\hat{R}_{\gamma}^{q \alpha_{0}}\left(\mathcal{N}_{s c}\right)=\frac{\sum_{m=0}^{\mathcal{M}_{s}\left(\mathcal{N}_{s c}+D\right)-1} y(m) y^{*}\left(m+\mathcal{N}_{s c}\right) e^{-2 i \pi m q \alpha_{0}}}{\mathcal{M}_{s}\left(\mathcal{N}_{s c}+D\right)} .
$$

$N_{c}$ represents the number of considered cycle frequencies to estimate the signal power. The choice of $N_{c}$ is a trade-off between the estimator bias and variance. In [13], we show that we must choose $q \alpha_{0}$ within the coherence bandwidth of the channel $B_{c}$. As the channel impulse response is unknown at reception, $B_{c}$ is approximated as $\hat{B}_{c}=1 /(\rho \hat{L})$ where $\rho$ is a coefficient expressing the desired correlation rate within $B_{c}$. Consequently, we choose $N_{c}=\min \left(\frac{\mathcal{N}_{s c}+D}{\rho \hat{L}}, \frac{\mathcal{N}_{s c}}{2 D}\right)$. As shown in [13], $\rho$ 's choice has only a very little influence on the estimator performance. The signal power C.C is estimated to be $\mathcal{O}\left(N_{c} \mathcal{M}_{s}\left(\mathcal{N}_{s c}+D\right)\right)$.

OFDM synchronization can be performed in a nondata-aided context by the mean of algorithms such as [16] and [17] for instance. The complexity of these algorithms is $\mathcal{O}\left(\mathcal{M}_{s} .\left(\mathcal{N}_{s c}+D\right) . D\right)$ for [16] and $\mathcal{O}\left(\mathcal{M}_{s} \cdot\left(\mathcal{N}_{s c}+D\right) \cdot D^{2}\right)$ for [17]. Miss-synchronization only impacts the noise variance estimator and has the following effects. If the symbol synchronization is not well performed, signal samples may be included in the noise variance estimator, leading to an overestimation of the noise variance. If the carrier frequency offset is not well mitigated, the phase of $\gamma\left(k\left(\mathcal{N}_{s c}+D\right)+m\right)$ and $\gamma\left(k\left(\mathcal{N}_{s c}+D\right)+\mathcal{N}_{s c}+m\right)$ will be different so that the redundancy induced by the $\mathrm{CP}$ will not be well exploited, leading once again to an overestimation of the noise variance. To put it in a nutshell, both events will lead to an underestimation of the signal-to-noise ratio, which is not so dramatic for the vertical handover process. Indeed, underestimating the SNR and not 
connecting to the access point are much better than overestimating it, and then we find that the QoS does not satisfy our needs and wasting time again finding other potential candidates. We point out that the method presented in [14], as our method, also requires a perfect time-frequency synchronization.

\subsection{Channel occupancy rate estimation}

In $[12,18]$, it has been highlighted that the usage of the channel bandwidth in a CSMA/CA system such as $\mathrm{WiFi}$ can be approximated as the ratio between the time in which the channel status is busy according to the NAV (network allocation vector) settings and the considered time interval. Indeed, prior to transmitting a frame, a station computes the amount of time necessary to send the frame based on the frame's length and data rate. This value is placed in the duration field in the header of the frame. By reading this file, we have access to the traffic load. The higher the traffic, the larger the NAV busy occupation, and vice versa. Then, once we read a NAV value during a certain time window, the available bandwidth and access delay can be estimated given a certain packet length [19]. The main drawback with this method is that it requires to be connected to the access point in order to have access to the NAV duration from the header. This may increase the decision time if many standards or access points (AP) are detected.

In this section, we propose a method that requires no connection to the AP and no NAV duration reading. This method [20] is based on a physical layer sensing: Considering that the medium is free when only noise is observed and occupied when signal plus noise samples are observed (data frame), we use a likelihood function that can distinguish the signal plus noise samples from the one corresponding to noise only. Once we get the number of signal plus noise samples, a simple ratio processing provides the network occupancy rate.

\subsubsection{Model structure}

In this section, we assume that CSMA/CA-based access points are detected. Between two consecutive frames we have different inter frame spacing (IFS) intervals, which guarantee different types of priority. At the receiver side, the observed signal is a succession of frames of noise samples corresponding to the IFS intervals or idle periods and of data frames (Figure 1).

For clarity reason, we assume in this section that we have only one data frame in the observation duration ( $N_{s}$ samples), and Section 2.2.2 explains the proposed algorithm to locate it.

Consider that our receiver is doted of $N$ antennas ${ }^{\mathrm{b}}$, and let $\mathbf{y}_{i}=\left[y_{i}(1), \ldots, y_{i}\left(N_{s}\right)\right]$ be a set of $N_{s}$ observations on the $i$ th antenna such that

$$
\begin{cases}y_{i}(m)=w_{i}(m) & 1 \leq m \leq m_{1}-1 \\ y_{i}(m)=\sum_{l=0}^{L_{i}-1} h_{i}(l) x\left(m-m_{1}-l\right)+w_{i}(m) & m_{1} \leq m \leq m_{2} \\ y_{i}(m)=w_{i}(m) & m_{2}+1 \leq m \leq N_{s}\end{cases}
$$

where the $x(m)$ is an OFDM source signal expressed as in $(1), h_{i}(l)$ is the channel response from source signal to the $i$ th antenna, and $L_{i}$ is the order of the channel $h_{i}$. The process $w_{i}(m)$ is a complex additive white Gaussian noise with zero mean and variance $\sigma_{w}^{2}$. The variance $\sigma_{w}^{2}$ is assumed to be known or at least estimated by a subspace-based algorithm [21], where multiple antennas at reception are required.

\subsubsection{Frame localization}

As presented in the previous section, the vector $\mathbf{y}_{i}$ can be divided into three parts: noise, signal plus noise, and noise. Starting from the set of observation $\mathbf{y}_{i}$, we would like to find which samples correspond to noise and which ones correspond to signal plus noise. This problem is a classical signal detection problem. Signal detection theory is a well-known problem in signal processing. This problem deals with the detectability of signals from noise. Many works have been done in this field, and a large literature exists $([22-24], \ldots)$. A maximum a posteriori testing, a Bayes criterion, a Neyman Pearson, or an energy detector [25] can be used. Here, we use another approach, since the samples are supposed to be independent in the noise areas and correlated in the signal plus noise area due to the channel effect and their OFDM structure. We propose to use a likelihood function that provides an information about the independence of the processed sample, and we are seeing later that this approach is close to a constant false alarm rate detector, when its main advantage relies




on the fact that it does not need to set a threshold value to the detector.

Let now $\mathbf{Y}_{i}(u)$ denotes the following set of observations:

$$
\mathbf{Y}_{i}(u)=\left[y_{i}(u), \ldots, y_{i}\left(N_{s}\right)\right] \quad 1 \leq u<N_{s}
$$

And let us define $f_{Y}$ the joint probability density function of $\mathbf{Y}_{i}(u)$. If $\mathbf{Y}_{i}(u)$ is composed of only noise samples

$$
f_{Y}\left(\mathbf{Y}_{i}(u)\right)=\prod_{m=u}^{N_{s}} f_{w}\left(y_{i}(m)\right),
$$

where $f_{w}$ is the probability density function of a complex normal law centered and variance $\sigma_{w}^{2}$, given by

$$
f_{w}(x)=\frac{1}{\pi \sigma_{w}^{2}} e^{-|x|^{2} / \sigma_{w}^{2}},
$$

The log-likelihood that the vector $\mathbf{Y}_{i}(u)$ is formed of $\left(N_{s}-u\right)$ noise-independent samples is expressed as

$$
\mathcal{L}_{i}(u)=\log \left[\prod_{m=u}^{N_{s}} f_{w}\left(y_{i}(m)\right)\right]
$$

Computing the mean of the $N$ log-likelihood functions expressed on each sensor, we get a criterion $\mathcal{J}(u)$ to provide an information about the nature of the processed samples

$$
\begin{aligned}
\mathcal{J}(u) & =\frac{1}{N} \sum_{i=1}^{N} \mathcal{L}_{i}(u) \\
& =-\left(N_{s}-u\right) \log \left(\pi \sigma_{w}^{2}\right)-\frac{1}{N \sigma_{w}^{2}} \sum_{i=1}^{N} \sum_{m=u}^{N_{s}}\left|y_{i}(m)\right|^{2}
\end{aligned}
$$

As $u$ varies in the interval $\left[1, m_{1}\right)$, the number of noise samples composing $\mathbf{Y}_{i}(u)$ decreases and so does $\mathcal{J}(u)$ until it reaches a minimum bound at $m_{1}$ (see Figure 2).

However, for $u$ varying from $m_{1}$ to $m_{2}$, the number of signal plus noise samples decreases; therefore, the ratio of noise samples to signal plus noise samples increases and by the way $\mathcal{J}(u)$ increases. It reaches its maximum value if and only if $\mathbf{Y}_{i}(u)$ contains only noise samples, i. e., when $u=m_{2}$.

Finally, for $m_{2}<u<N_{s}, \mathcal{J}(u)$ decreases again for the same reason that the one explained for $1<u<m_{1}$.

We conclude that the edges of the detected frame can be estimated as

$$
\left\{\begin{array}{l}
\hat{m}_{1}=\arg \min _{u}[\mathcal{J}(u)] \\
\hat{m}_{2}=\arg \max _{u}[\mathcal{J}(u)]
\end{array}\right.
$$

\subsubsection{Estimation of the channel occupancy rate}

When we have only one data frame in the observed window, the occupancy rate can easily be estimated
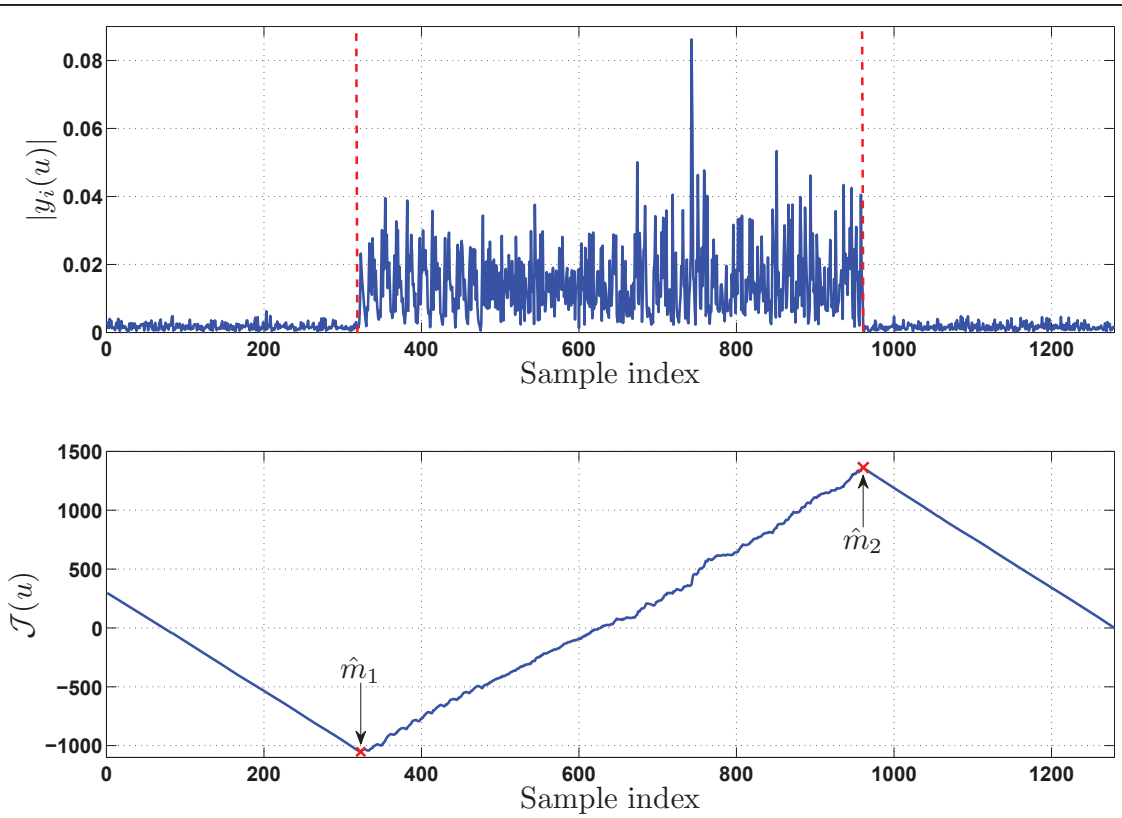

Figure 2 Example with one frame and corresponding criterion behavior. 
thanks to the previous criterion by $\frac{\hat{m}_{2}-\hat{m}_{1}}{N_{s}}$. However, the assumption to have only one frame in the observation window is too restrictive. In practice, we may get a signal as shown in Figure 3 or with more frames.

Based on the behavior of $\mathcal{J}(u)$, we can clearly see (Figure $3 \mathrm{~b})$ that the slope of $\mathcal{J}(u)$ is positive when $u$ corresponds to the index of a signal plus noise sample and negative when $u$ corresponds to the index of a noise sample. Therefore, we can take advantage of the gradient of $\mathcal{J}(u)$ to distinguish the nature of the observed samples. Introducing the function $\Phi(u)$ such that

$$
\Phi(u)=\frac{1}{2}[\operatorname{sign}\{\nabla(\mathcal{J}(u))\}+1] .
$$

Here, we denote by $\nabla$ the gradient of $\mathcal{J}(u)$ processed using the central difference method, such that the derivative for any point of index $u \notin\left\{1, N_{s}\right\}$ is processed as

$$
\nabla(\mathcal{J}(u))=\frac{1}{2}(\mathcal{J}(u+1)-\mathcal{J}(u-1)) .
$$

For the first point, we use the forward finite difference such that

$$
\nabla(\mathcal{J}(1))=\mathcal{J}(2)-\mathcal{J}(1) .
$$

Finally, at the right end element, a backward difference is used

$$
\nabla\left(\mathcal{J}\left(N_{s}\right)\right)=\mathcal{J}\left(N_{s}\right)-\mathcal{J}\left(N_{s}-1\right)
$$

sign \{.\} denotes the sign operator. According to this, $\Phi$ $(u)$ equals 1 when signal plus noise samples are present and zero when it is only noise, and the channel occupancy rate is estimated by

$$
\widehat{C_{o r}}=\frac{1}{N_{s}} \sum_{u=1}^{N_{s}} \Phi(u)
$$

\subsubsection{Criterion validation limits}

In this section, we propose to investigate the limits of the proposed criterion $\mathcal{J}(u)$. The aim is to find the dynamic where $\mathcal{J}(u)$ well behaves, i.e., where its slope is positive for signal plus noise samples and negative for noise samples.

- For $1 \leq u \leq m_{1}$ : $\mathcal{J}(u)$ decreases only if $\frac{\partial \mathbb{E}[\mathcal{J}(u)]}{\partial u}<0$, and therefore if

$\mathbb{E}[\mathcal{J}(u)]=-\left(N_{s}-u\right) \log \left(\pi \sigma_{w}^{2}\right)-\frac{1}{\sigma_{w}^{2}}\left[\left(m_{1}-u\right) \sigma_{w}^{2}+\left(m_{2}-m_{1}\right)\left(\sigma_{w}^{2}+S\right)+\left(N_{s}-m_{2}\right) \sigma_{w}^{2}\right]$

the derivative costs: $\frac{\partial \mathbb{E}[\mathcal{J}(u)]}{\partial u}=\log \left(\pi \sigma_{w}^{2}\right)+1$, and we get

$\sigma_{w}^{2}<\frac{1}{\pi e}$

- For $m_{1} \leq u \leq m_{2}: \mathcal{J}(u)$ is an increasing function only if $\frac{\partial \mathbb{E}[\mathcal{J}(u)]}{\partial u}>0$, then if

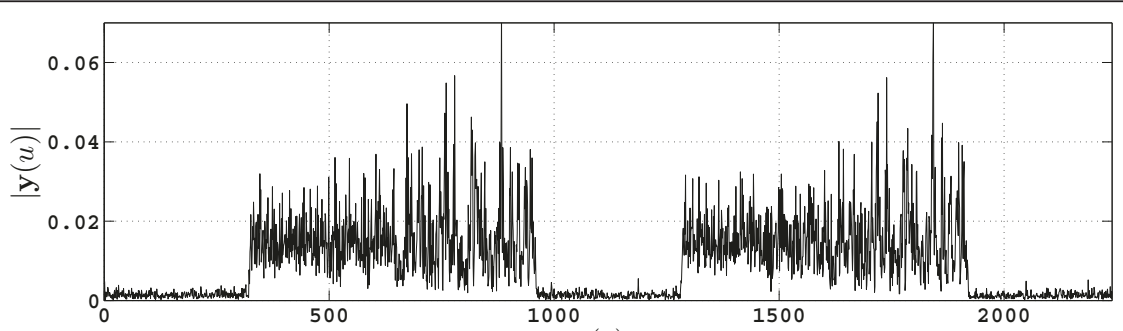

(a)



(b)

Figure 3 (a) Absolute value of a wifi signal, (b) corresponding behavior of the criterion $\mathcal{J}(u)$ 


$$
\mathbb{E}[\mathcal{J}(u)]=-\left(N_{s}-u\right) \log \left(\pi \sigma_{w}^{2}\right)-\frac{1}{\sigma_{w}^{2}}\left[\left(m_{2}-u\right)\left(\sigma_{w}^{2}+S\right)+\left(N_{s}-m_{2}\right) \sigma_{w}^{2}\right]
$$

the partial derivative is

$$
\frac{\partial \mathbb{E}[\mathcal{J}(u)]}{\partial u}=\log \left(\pi \sigma_{w}^{2}\right)+\frac{1}{\sigma_{w}^{2}}\left(\sigma_{w}^{2}+S\right),
$$

and $\mathcal{J}(u)$ increases only if

$$
\sigma_{w}^{2}>\frac{1}{\pi e^{(1+\gamma)}}
$$

where $\gamma=\frac{S}{\sigma_{w}^{2}}$ is the signal-to-noise ratio.

- For $m_{2} \leq u \leq N_{s}$ : we get the same result as in (16).

As a conclusion for an optimal behavior of $\mathcal{J}(u)$, the noise variance must satisfy

$$
\frac{1}{\pi e^{(1+\gamma)}}<\sigma_{w}^{2}<\frac{1}{\pi e} .
$$

This inequality represents the limits of the proposed criterion. It means that the performance of the proposed method depends on the noise variance value and also on the signal-to-noise ratio. Therefore, if the noise variance does not satisfy Equation (19), we can think to adjust it applying a certain gain on the received signal. Indeed, by multiplying the whole vector of observation $\mathbf{y}$ by a gain $\sqrt{\eta}$, the noise variance is no longer $\sigma_{w}^{2}$ but $\eta \sigma_{w}^{2}$, where $\eta$ must be chosen such that it satisfies

$$
\frac{1}{\pi e^{1+\gamma}}<\eta \sigma_{w}^{2}<\frac{1}{\pi e}
$$

The right part of the inequality is easy to satisfy, but unfortunately the left part requires the knowledge of the signal-to-noise ratio, which is not available in our case. Another approach is to introduce a new criterion that overcomes this drawback; this criterion is the distance between $\mathcal{J}(u)$, a Parzen estimator-based criterion introduced in the next section.

\subsubsection{Parzen estimator-based criterion}

The proposed solution consists in processing a new criterion that aims to minimize the distance between the true probability density function of the noise and a Parzen-estimated probability density function of the observed samples [26,27]. The main advantage of this new criterion is that it does not rely on Equation (19). We see in Section 5.1 that its performance remains constant for any value of $\sigma_{w}^{2}$.
Starting from the set of observations

$$
\Xi=\left\{\Re\left\{y_{i}(m)\right\}, \Im\left\{y_{i}(m)\right\}\right\}, i \in\{1, \ldots, N\}, m \in\left\{1, \ldots, N_{s}\right\}(21)
$$

where $\mathfrak{R}\{$.$\} and \mathfrak{\Im}\{$.$\} denotes the real and imaginary$ part of the sample. We get $2 N N_{s}$ samples available for estimating the Parzen window density distribution. Given a sample $y_{i}(m)=p_{i}(m)+j \cdot q_{i}(m)$, its Parzen window distribution is given by

$$
\hat{f}\left(\gamma_{i}(m)\right)=\hat{f}\left(p_{i}(m)\right) \hat{f}\left(q_{i}(m)\right),
$$

where

$$
\hat{f}(z)=\frac{1}{2 N N_{s} F} \sum_{k=0}^{2 N N_{s}-1} K\left(\frac{z-z_{k}}{F}\right) .
$$

Such that $K$ is the Parzen window kernel and $F$ is a smoothing parameter called the bandwidth. This kernel has to be a suitable p.d.f function. We use Gaussian kernels with standard deviation one. The new processed criterion is

$$
\mathcal{J}_{K}(u)=\frac{1}{N} \sum_{i=1}^{N} \log \left[\prod_{m=u}^{N_{s}} \hat{f}\left(y_{i}(m)\right)\right] .
$$

Once we get $\mathcal{J}_{K}(u)$, we measure the distance between $\mathcal{J}(u)$ and $\mathcal{J}_{K}(u)$ to obtain a new criterion

$$
\mathcal{K}(u)=\left|\mathcal{J}(u)-\mathcal{J}_{K}(u)\right| .
$$

Substituting $\mathcal{J}(u)$ by $\mathcal{K}(u)$ in Equation 14, the function $\Phi(u)$ is processed to be then used to find the channel occupancy rate Equation (15).

\subsubsection{Fluctuations problem}

The difficulty is to estimate the channel occupancy rate accurately for low signal-to-noise ratio. In fact, there are fluctuations that can mislead the decision for a given sample (Figure 4). To fix this problem, we propose to use a smoothing technique.

The choice of the length of the smoothing window $W$ is very important. We choose $W$ equal to the length of a SIFS (for Short IFS), which is the smallest interframe interval. Thus, theoretically, we can not get a set of successive noise samples of a length less than a SIFS. Then, if we met a set of noise-only samples of length less than an SIFS, it means that the algorithm took the wrong decision and $\Phi(u)$ will be forced to 1 for those samples.

\subsubsection{Relation with the CFAR method}

We can demonstrate that there is a direct relation between our method and the CFAR (Constant False Alarm Rate [28]) method. The main difference of the proposed technique is that it does not rely on a false alarm probability $P_{f a}$. Indeed, the proposed approach only depends on the noise variance value. 




(a)



(b)

Figure 4 (a) $\mathcal{J}(u)$, (b) corresponding $\Phi(u)$.

First of all, let us consider the case of the Gaussian noise. The CFAR approach relies on a threshold associated with a false alarm $P_{f a}$. Considering the following hypothesis test

$$
\left\{\begin{array}{l}
H_{0}: y_{i}(m)=w_{i}(m) \\
H_{1}: y_{i}(m)=\sum_{l=0}^{L_{i}-1} h_{i}(l) x_{j}(m-l)+w_{i}(m)
\end{array}\right.
$$

and a given threshold $\lambda$, the probability of false alarm can be expressed as

$$
P_{f a}=\operatorname{Pr}\left\{\left|y_{i}(m)\right|^{2} \geq \lambda \mid H_{0}\right\} .
$$

Since the noise is supposed Gaussian, its absolute value follows a Rayleigh distribution $\mathcal{R}\left(\frac{\sigma_{w}}{\sqrt{2}}\right)$ and

$$
\begin{aligned}
P_{f a} & =2 \int_{\lambda}^{\infty} \frac{y_{i}(m)}{\sigma_{w}^{2}} \exp \left(-\frac{y_{i}(m)^{2}}{\sigma_{w}^{2}}\right) \mathrm{d} y_{i}(m), \\
& =\exp \left(-\frac{\lambda^{2}}{\sigma_{w}^{2}}\right) .
\end{aligned}
$$

Therefore, an observed sample is considered as signal plus noise sample if and only if

$$
\left|\gamma_{i}(m)\right|^{2}>-\sigma_{w}^{2} \log \left(P_{f a}\right) .
$$

In our case, considering that $\nabla\left(\mathcal{L}_{i}(m)\right)=\mathcal{L}_{i}(m+1)-\mathcal{L}_{i}(m)$, we have the following expression

$$
\nabla\left(\mathcal{L}_{i}(m)\right)=\log \left(\pi \sigma_{w}^{2}\right)+\frac{1}{\sigma_{w}^{2}}\left|\gamma_{i}(m)\right|^{2} .
$$

As said previously, the symbols are considered as signal plus noise if and only if the gradient is positive. It follows that

$$
\left|y_{i}(m)\right|^{2}>-\sigma_{w}^{2} \log \left(\pi \sigma_{w}^{2}\right) .
$$

We obtain the same criteria with the CFAR if we choose a $P_{f a}=\pi \sigma_{w}^{2}$, providing that Equation (19) is satisfied. The main advantage of the proposed approach relies on the fact that the choice of the $P_{f a}$ is automatic and achieves good performance when Equation (19) is satisfied.

As there is a recursive relation between two consecutive samples of $\mathcal{J}(u)$, such that

$$
\mathcal{J}(u-1)=\mathcal{J}(u)-\left(\log \left(\pi \sigma_{w}^{2}\right)-\frac{1}{N \sigma_{w}^{2}} \sum_{i=1}^{N}\left|\gamma_{i}(u)\right|^{2}\right) .
$$

To reduce the computational cost, we propose to compute the criterion in the backward sense, i.e., from its last element and then deducing the other elements recursively. In this case, the CC is reduced to $\mathcal{O}\left(N N_{s}\right)$. The whole algorithm is described in Algorithm 1.

Algorithm 1 Channel Occupancy Rate Estimation Observe $N_{s}$ samples on the desired channel;

$\mathcal{J}\left(N_{s}\right)=-\frac{1}{N \sigma_{w}^{2}} \sum_{i=1}^{N}\left|y_{i}\left(N_{s}\right)\right|^{2} ;$


$\left.\operatorname{do} \mathcal{J}(u)=\mathcal{J}(u+1)-\left(\log \left(\pi \sigma_{w}^{2}\right)+\frac{1}{N \sigma_{w}^{2}} \sum_{i=1}^{N}\left|y_{i}(u)\right|^{2}\right)\right)$

end for

Compute the functions $\Phi(u)$ values using (14); 
Smooth $\Phi(u)$ thanks to the described procedure in 2.2.6;

Deduce the $C_{\text {or }}$ thanks to (15).

As the number of users increases, the load increases and the collision probability too. To maintain a good QoS and to avoid the collisions, the backoff intervals are increased in an exponential manner. This leads to injecting a large amount of white spaces in the communication exchange For congested networks, i.e., where all the nodes have a frame ready to be sent in their buffers, we remark that the channel occupancy rate decreases. In order to avoid a VHO in that particular case, it is relevant to have access to another relevant metric in such situation, which is the collision rate.

\subsection{Frame collision detection}

The contention-based access mechanism in WiFi implies that all the stations have to listen to the channel before competing for the access in order to avoid collision between the frames. Unfortunately, as the number of competing stations increases, the collision probability increases and the throughput decreases affecting the QoS. Then, the collision rate is a good metric for both horizontal handover where many access points are available and also vertical handover if we wish to hand off from any standard to an OFDM access point.

A proposed method $[29,30]$ for collision detection in a WiFi system suggests that the AP of a basic service set (BSS) measures RF energy duration on the channel and broadcasts this result. Then, stations can detect collisions by checking the duration against their previous transmission schedules, if they are different it means that a collision occurs. This method assumes that the mobile is able to measure this time duration and requires to be connected and synchronized with the access point.

Within this framework, we propose a method for collision detection that requires no connection to the AP. Once the data frames are detected thanks to the algorithm presented in Section 2.2.2, we use an information theoretic criterion to get the rank of the autocorrelation matrix of the observed frame.

Unfortunately, to estimate the number of sources, the channel length is necessary. To skip this step, we propose to exploit the OFDM structure of the signals: since the channel length is always less than the cyclic prefix, using a smoothing window for the autocorrelation matrix of a length equal to the cyclic prefix, we can get the number of sources and decide whether a collision occurred or not (number of sources greater than 1). In this case, the number of antennas must be greater than the number of source, so we need at least 3 antennas to detect the collision. The signal model is said to be
MIMO for multiple input multiple output. We consider that $M$ sources are emitting and that the receiver is doted of $N$ antennas. The observed signal on the $i$ th antenna is expressed as

$$
y_{i}(m)=\sum_{j=1}^{M} \sum_{l=0}^{L_{i j}-1} h_{i j}(l) x_{j}(n-l)+w_{i}(m),
$$

where the $x_{j}(m)$ for $j=1, \ldots, M$ are OFDM source signals expressed as in (1), $h_{i j}(l)$ is the channel impulse response from source signal $j$ to the $i$ th antenna, and $L_{i j}$ is the order of the channel $h_{i j}$.

Consider that we detected a data frame of length $N_{f}$, and let $L_{j}=\max _{i}\left(L_{i j}\right)$ be the longest impulse response of the channel, zero-padding $h_{i j}(l)$ if necessary. First, defining the following vectors

$$
\begin{aligned}
& \mathbf{y}(m)=\left[\gamma_{1}(m), \gamma_{2}(m), \ldots, \gamma_{N}(m)\right]^{T}, \\
& \mathbf{h}_{j}(m)=\left[h_{1 j}(m), h_{2 j}(m), \ldots, h_{N j}(m)\right]^{T}, \\
& \mathbf{w}(m)=\left[w_{1}(m), w_{2}(m), \ldots, w_{N}(m)\right]^{T},
\end{aligned}
$$

we can express the signal model as

$$
\mathbf{y}(m)=\sum_{j=1}^{M} \sum_{l=0}^{L_{j}-1} \mathbf{h}_{j}(l) x_{j}(m-l)+\mathbf{w}(m),
$$

Considering an observation window of $d$ samples and defining

$$
\begin{aligned}
\mathbf{y}_{d}(m)= & {\left[\mathbf{y}^{T}(m), \ldots, \mathbf{y}^{T}(m-d+1)\right]^{T}, } \\
\mathbf{x}_{d}(m)= & {\left[x_{1}(m), \ldots, x_{1}(m-d-L+1), \ldots,\right.} \\
& \left.x_{M}(m), \ldots, x_{M}(m-L-d+1)\right]^{T}, \\
\mathbf{w}_{d}(m)= & {\left[\mathbf{w}^{T}(m), \ldots, \mathbf{w}^{T}(m-d+1)\right]^{T}, }
\end{aligned}
$$

we get

$$
\mathbf{y}_{d}(m)=\mathcal{H} \mathbf{x}_{d}(m)+\mathbf{w}_{d}(m),
$$

where $\mathcal{H}$ is $N d \times(L+M d)\left(L \stackrel{\text { def }}{=} \sum_{1}^{M} L_{j}\right)$ Sylvester matrix defined as

$$
\begin{aligned}
& \mathcal{H}=\left[\mathcal{H}_{1}, \mathcal{H}_{2}, \ldots, \mathcal{H}_{M}\right],
\end{aligned}
$$

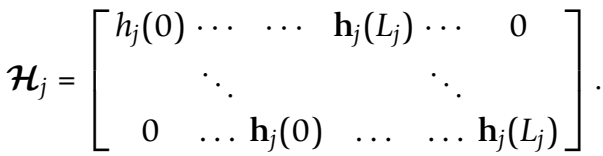

Note that the dimension of $\mathcal{H}_{j}$ is $N d \times\left(L_{j}+d\right)$. 
Defining the statistical covariance matrices of the signals and noise as

$$
\begin{aligned}
& \mathbf{R}_{\gamma}=\mathbb{E}\left[\mathbf{y}_{d}(m) \mathbf{y}_{d}(m)^{H}\right], \\
& \mathbf{R}_{x}=\mathbb{E}\left[\mathbf{x}_{d}(m) \mathbf{x}_{d}(m)^{H}\right], \\
& \mathbf{R}_{w}=\mathbb{E}\left[\mathbf{w}_{d}(m) \mathbf{w}_{d}(m)^{H}\right],
\end{aligned}
$$

we have the following relation

$$
\mathbf{R}_{y}=\mathcal{H} \mathbf{R}_{x} \mathcal{H}^{H}+\sigma_{w}^{2} \mathbf{I}_{N d}
$$

where $\mathbf{I}_{N d}$ is the identity matrix of order $N d$ and (.) ${ }^{H}$ is the transpose conjugate operator.

Assuming that the channels have no common zeros, and for a large enough observation window of a size $d$, we establish that the rank of $\mathbf{R}_{x}$ is

$$
r=\min \{(M d+L), d N\}
$$

Using an information theoretic criterion, like AIC or MDL [31], it is possible to get an estimate of $r$, such that

$$
\begin{aligned}
& \operatorname{AIC}(k)=-2 \log \left(\frac{\prod_{i=k+1}^{N d} \lambda_{i}^{1 /(N d-k)}}{\frac{1}{N d-k} \sum_{i=k+1}^{N d} \lambda_{i}}\right)^{(N d-k) N_{f}}+2 k(2 N d-k), \\
& \operatorname{MDL}(k)=-\log \left(\frac{\prod_{i=k+1}^{N d} \lambda_{i}^{1 /(N d-k)}}{\frac{1}{N d-k} \sum_{i=k+1}^{N d} \lambda_{i}}\right)^{(N d-k) N_{f}}+\frac{k}{2}(2 N d-k) \log N_{f},
\end{aligned}
$$

where the $\lambda_{i}$ for $i=1, \ldots, N d$ are the sorted eigenvalues of $\mathbf{R}_{y}, N_{f}$ represents the length of the detected frame. The rank of the autocorrelation matrix $\mathbf{R}_{y} \hat{r}$ is determined as the value of $k \in\{0, \ldots, N d-1\}$ for which either the AIC or the MDL is minimized.

$$
\left\{\begin{array}{l}
\hat{r}_{\mathrm{AIC}}=\arg \min _{k}[\mathrm{AIC}] \\
\hat{r}_{\mathrm{MDL}}=\arg \min _{k}[M D L]
\end{array}\right.
$$

Therefore, according to Equation (44), the number of sources $M$ is estimated as the nearest integer to $\frac{r-L}{d}$. Unfortunately, the channel length $L$ is unknown, and we should have it to estimate $M$.

To avoid this step, we propose to exploit the properties of the OFDM signals. We know that the length of the cyclic prefix is always chosen to be greater than $L_{i j}$. So, if the smoothing factor $d$ is defined as equal to the cyclic prefix, we are sure that $L_{i j}<d$.
We can generalize that to estimate a number of sources greater than one. In fact, if $r=M d+L$ then $L$ $=r-M d$. Since $L=\sum_{j=1}^{M} \max _{i}\left(L_{i j}\right)$, we are sure that $L<$ $M d$ and by the way $r-M d<M d$. Thus, $r / M<2 d$, and therefore $M>\frac{r}{2 d}$. We conclude that $\hat{M}$ is the nearest integer greater than $\frac{r}{2 d}$. If this value equals 1 , it means that there is indeed one source, otherwise more than one source is present and a collision occurs. The algorithm is described in Algorithm 2. For each frame, we have to compute the eigenvalue decomposition (EVD) and then perform AIC or MDL. As the C.C of these two algorithms is negligible compared to the EVD, the computational cost is proportional to an EVD.

Algorithm 2 Collision detection algorithm

nb_collision $=0$;

Run algorithm described in Section 2.2.2;

for each detected data frame do

Process the autocorrelation matrix $\mathbf{R}_{y}$;

Compute $r$ thanks to (45) or (46);

if $\operatorname{ceil}(r / 2 d)>1$ then

nb_collision $=$ nb_collision +1 ;

end if

end for

collision rate $=\frac{\text { nb_collision }}{\text { the number of detected frames }}$

\section{Metrics for OFDMA-based networks}

Orthogonal frequency division multiple access (OFDMA) is a multi-access technique based on orthogonal frequency division multiplexing (OFDM) digital modulation scheme. Multiple access is achieved in OFDMA by assigning subsets of subcarriers to individual users in a given time slot. This technique allows to support differentiated quality of service (QoS), i.e., to control the data rate and error probability individually for each user.

First, we propose to apply the algorithm presented in Section 2.1 to get an estimate of the downlink SNR in an OFDMA-based network. Then, we propose an alternative approach to estimate the time frequency activity rate, which is a similar metric of the channel occupancy rate for CSMA/CA-based systems. Concerning the collision rate, as said previously, since OFDMA-based systems are full duplex, no collision occurs and it has no meaning as a metric.

\subsection{SNR estimation for OFDMA based systems}

Assuming that an OFDMA symbol consists of up to $\mathcal{N}_{s c}$ active subcarriers, we can modify Equation (1) to get the expression of an OFDMA signal

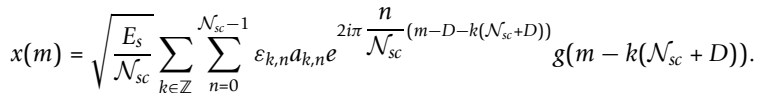


In this case, $\varepsilon_{k, n}$ is a set of i.i.d random variable valued in $\{0,1\}$, expressing the absence or presence of signal activity in the $(k, n)$ time frequency slot. The received signal is expressed as in Equation (2), and the SNR is expressed as

$$
\mathrm{SNR}=\frac{S}{\sigma_{w}^{2}}
$$

with

$$
S=E_{s} \mathbb{E}\left[\left|\varepsilon_{k, n} a_{k, n}\right|^{2}\right] \sum_{l=0}^{L-1} \sigma_{h(l)}^{2} .
$$

The whole algorithm presented in Section 2.1 stays valid for OFDMA signals.

\subsection{Time-frequency activity rate estimation for OFDMA system}

In OFDMA-based systems, when the number of active subcarriers is small, the data traffic should also be. Therefore, providing a satisfying downlink signal strength, it is better for a multi-mode terminal to connect on such a base station rather than on one where the data traffic is high (high number of active subcarrier).

In this section, we focus on the passive estimation of the allocation rate of OFDMA physical channels' timefrequency slots. The allocation rate is defined as the number of active slots (allocated symbols) divided by the total number of slots per frame.

In some networks such as WiMAX, the physical channels' allocation rate is regularly broadcasted by the base station so that it can be known by any terminal. However, this requires a multi-mode terminal that listens to the surrounding networks to intercept every frame preamble. If the multi-mode terminal has to decode every intercepted preamble to get this information, the vertical handover can be a very time- and power-consuming process.

An alternative approach developed in this section is to get the OFDMA physical channels' allocation rate by blindly estimating the time-frequency activity rate of OFDMA physical signals. Such approach focuses on the signal properties and therefore does not require any message decoding (assuming this message is made available by the base station, which may not be the case in all OFDMA networks). To the best of our knowledge, there is no algorithm published to date that addresses the blind estimation of the time-frequency activity rate of OFDMA signals. We propose a method [32] with a low computational cost to estimate the time frequency activity rate of a WiMAX networks. This method is based on the estimation of the first- and second-order moments of the received signal.
The received signal is expressed as in Equation (2). We assume that the receiver is synchronized with the transmitter in time and in frequency. This synchronization can be realized thanks to the frame preamble or thanks to blind techniques presented in [16] and [33]. We also assume that the noise power $\sigma_{w}^{2}$ is known or at least estimated thanks to blind methods such as those detailed in Section 2.1 or in [13,34].

\subsubsection{Estimation algorithm}

The estimation of the time-frequency activity rate $\tau$ is equivalent to detect the active slots from the non-active ones

$$
\tau=\frac{\sum_{k, n} I\left(\varepsilon_{k, n}=1\right)}{\mathcal{M}_{s} \mathcal{N}_{s c}},
$$

where $I(A)$ is the indicator function of any event $A$ and $\mathcal{M}_{s}$ is the number of observed OFDM symbols. Intuitively, considering that $\sigma_{w}^{2}$ is known, a classic detector structure could be used so that

$$
\hat{\tau}=\frac{\sum_{k, n} I\left(\left|Y_{k, n}\right|>\theta\left(\sigma_{w}\right)\right)}{\mathcal{M}_{s} \mathcal{N}_{s c}},
$$

where $\theta\left(\sigma_{w}\right)$ is a threshold function and $Y_{k, n}$ is the signal observation on the slot $(k, n)$.

$$
\begin{aligned}
& Y_{k, n} \triangleq \frac{1}{\mathcal{N}_{s c}} \sum_{m=0}^{\mathcal{N}_{s c}-1} \gamma\left[k\left(\mathcal{N}_{s c}+D\right)+D+m\right] e^{-2 i \pi \frac{n m}{\mathcal{N}_{s c}},} \\
& =\varepsilon_{k, n} a_{k, n} H_{k, n} \sqrt{E_{s}}+W_{k, n,}
\end{aligned}
$$

where $H_{k, n}$ and $W_{k, n}$ are, respectively, the channel frequency response at subcarrier $n$ and the noise at subcarrier $n$ of the $k$ th received symbol. The limitation of such approach is that the performance is strongly impacted by the choice of a threshold. In order to avoid this constraint, we hereafter propose complementary alternative method. The proposed technique relies on the absolute value of the first- and second-order moments of the observed samples. These moments are indeed dependent of the activity rate $\tau$.

For all $(k, n)$ such that $\varepsilon_{k, n}=0$, the observations are made of noise-only slots such that they satisfy $Y_{k, n} \sim \mathcal{C N}\left(0, \sigma_{w}^{2}\right)$. Therefore, in this case the absolute value $\left|Y_{k, n}\right|$ has a Rayleigh distribution and its expectation is given by

$$
\mathbb{E}\left[\left|Y_{k, n}\right| / \varepsilon_{k, n}=0\right]=\frac{\sqrt{\pi}}{2} \sigma_{w}
$$

where $\mathbb{E}[. /$.$] defines the conditional expectation.$

When the observations are made of signal plus noise samples (i.e., $\varepsilon_{k, n}=1$ ), the distribution of $Y_{k, n}$ is hard to define. Indeed, actual systems are using the adaptive 
modulation and coding (AMC) scheme, and the constellation can be different from a slot to another. The $a_{k, n}$ may have a distribution corresponding to BPSK, QPSK, 16-QAM, or 64-QAM [35]. According to the principle of maximum entropy [36], the state of ignorance on the constellation distribution is here modeled by an uniform law. Hence, without prior information, we assume that the probability to get each constellation equals $1 / 4$. (Note that the impact of this assumption is discussed in Section 4). Consequently, the expectation of $\left|Y_{k, n}\right|$ when $\varepsilon_{k, n}=1$ can be written as

$$
\begin{aligned}
\mathbb{E}\left[\left|Y_{k, n}\right| / \varepsilon_{k, n}=1\right] & =\mathbb{E}\left[\left|a_{k, n} H_{k, n} \sqrt{E_{s}}+W_{k, n}\right|\right], \\
& =\frac{1}{4} \sum_{j=1}^{4} \mathbb{E}\left[\left|a_{k, n} H_{k, n} \sqrt{E_{s}}+W_{k, n}\right| / a_{k, n} \in \mathcal{C}_{M_{j}}\right],
\end{aligned}
$$

where the $C_{M_{j}}$ constellations are $M_{j}$-QAM such that for $j=1, \ldots, 4, M_{j}$ is equal to $2,4,16,64$.

Assuming a Gaussian noise, a Rayleigh fading channel and a known $a_{k, n}$, the distribution of the observed slots is Gaussian: $Y_{k, n} / a_{k, n}, \varepsilon_{k, n}=1 \sim \mathcal{C N}\left(0, \sum_{0}^{L-1} \sigma_{h(l)}^{2} E_{s}\left|a_{k, n}\right|^{2}+\sigma_{w}^{2}\right)$. It then follows that the absolute value $\left|Y_{k, n} / a_{k, n}, \varepsilon_{k, n}=1\right|$ has a Rayleigh distribution. After performing integration over all the possible values of $a_{k, n}$ in each $C_{M_{j}}$ constellation, we find that

$$
\mathbb{E}\left[\left|Y_{k, n}\right| / \varepsilon_{k, n}=1\right]=\frac{\sqrt{\pi}}{2} \frac{1}{4} \sum_{j=1}^{4} \frac{1}{M_{j}} \sum_{p=1}^{M_{j}} \sqrt{\sum_{l} \sigma_{h(l)}^{2} E_{s}\left|c_{p}\right|^{2}+\sigma_{w^{\prime}}^{2}}
$$

where $c_{p}$ is the $p$ th symbol of te constellation $C_{M_{j}}$, and consequently,

$$
\begin{aligned}
& \mathbb{E}\left[\left|Y_{k, n}\right| \mid \varepsilon_{k, n}=1\right]=\frac{\sqrt{\pi}}{8}\left[\frac{5}{2} \sqrt{\sum_{l} \sigma_{h(l)}^{2} E_{s}+\sigma_{w}^{2}}+\frac{1}{4} \sqrt{\sum_{l} \sigma_{h(l)}^{2} \frac{E_{s}}{5}+\sigma_{w}^{2}}\right. \\
& +\frac{1}{4} \sqrt{\frac{9}{5} \sum_{l} \sigma_{h(l)}^{2} E_{s}+\sigma_{w}^{2}}+\frac{1}{16} \sqrt{\sum_{l} \sigma_{h(l)}^{2} \frac{E_{s}}{21}+\sigma_{w}^{2}} \\
& +\frac{1}{8} \sqrt{\frac{5}{21} \sum_{l} \sigma_{h(l)}^{2} E_{s}+\sigma_{w}^{2}}+\frac{1}{16} \sqrt{\frac{3}{7} \sum_{l} \sigma_{h(l)}^{2} E_{s}+\sigma_{w}^{2}} \\
& +\frac{1}{8} \sqrt{\frac{13}{21} \sum_{l} \sigma_{h(l)}^{2} E_{s}+\sigma_{w}^{2}}+\frac{1}{8} \sqrt{\frac{17}{21} \sum_{l} \sigma_{h(l)}^{2} E_{s}+\sigma_{w}^{2}} \\
& +\frac{3}{16} \sqrt{\frac{25}{21} \sum_{l} \sigma_{h(l)}^{2} E_{s}+\sigma_{w}^{2}}+\frac{1}{8} \sqrt{\frac{29}{21} \sum_{l} \sigma_{h(l)}^{2} E_{s}+\sigma_{w}^{2}} \\
& \left.+\frac{13}{8} \sqrt{\frac{37}{21} \sum_{l} \sigma_{h(l)}^{2} E_{s}+\sigma_{w}^{2}}+\frac{13}{16} \sqrt{\frac{7}{3} \sum_{l} \sigma_{h(l)}^{2} E_{s}+\sigma_{w}^{2}}\right] . \\
& =\varphi\left(\sum_{l} \sigma_{h(l)}^{2} E_{s}\right) \text {. }
\end{aligned}
$$

where $\phi$ is a function that associate with each $\sum_{l} \sigma_{h(l)}^{2} E_{s}$ the expectation $\mathbb{E}\left[\left|Y_{k, n}\right| / \varepsilon_{k, n}=1\right]$, when $\sigma_{w}^{2}$ is assumed to be known.

Since $\tau \%$ of the slots are active and $(1-\tau) \%$ are not, the expectation of the module of the observed samples is expressed as

$$
\mathbb{E}\left[\left|Y_{k, n}\right|\right]=\tau \varphi\left(\sum_{l} \sigma_{h(l)}^{2} E_{s}\right)+(1-\tau) \frac{\sqrt{\pi}}{2} \sigma_{w} .
$$

Moreover, the second-order moment $\mathbb{E}\left[\left|Y_{k, n}\right|^{2}\right]$ is given by

$$
\mathbb{E}\left[\left|Y_{k, n}\right|^{2}\right]=\sigma_{w}^{2}+\tau \sum_{l} \sigma_{h(l)}^{2} E_{s,}, \forall \varepsilon_{k, n} .
$$

It follows that

$$
\sum_{l} \sigma_{h(l)}^{2} E_{s}=\frac{\mathbb{E}\left[\left|Y_{k, n}\right|^{2}\right]-\sigma_{w}^{2}}{\tau}
$$

If we denote by $\mu_{1}=\mathbb{E}\left[\left|Y_{k, n}\right|\right]$ and $\mu_{2}=\mathbb{E}\left[\left|Y_{k, n}\right|^{2}\right]$, then

$$
\begin{aligned}
& \hat{\mu}_{1}=\frac{1}{\mathcal{M}_{s} \mathcal{N}_{s c}} \sum_{k=0}^{M-1} \sum_{n=0}^{N-1}\left|Y_{k, n}\right|, \\
& \hat{\mu}_{2}=\frac{1}{\mathcal{M}_{s} \mathcal{N}_{s c}-1} \sum_{k=0}^{M-1} \sum_{n=0}^{N-1}\left|Y_{k, n}\right|^{2} .
\end{aligned}
$$

Substituting this value in Equation (58), an estimate of the channel occupancy rate $\hat{\tau}$ is obtained by solving the following equation

$$
\hat{\tau} \varphi\left(\frac{\hat{\mu}_{2}-\sigma_{w}^{2}}{\hat{\tau}}\right)+(1-\hat{\tau}) \frac{\sqrt{\pi}}{2} \sigma_{w}-\hat{\mu}_{1}=0 .
$$

This equation has no analytical solution. We propose to solve it by a binary search algorithm. The whole corresponding technique is presented in Algorithm 3. The computational cost of the proposed algorithm is negligible compared to the FFT, and thus the C.C is $\mathcal{O}\left(\mathcal{N}_{s c} \log \mathcal{N}_{s c}\right)$.

Algorithm 3 Moments method

Observe $\mathcal{M}_{s}$ OFDM symbols;

Estimate $\sigma_{w}^{2}$;

Compute $Y_{k, n}$;

Compute $\hat{\mu}_{1}$ and $\hat{\mu}_{2}$ thanks to (61) and (62);

Deduce $\hat{\tau}$ solving (63) thanks to the binary search algorithm.

\section{Architecture of the proposed detector}

The current design of cognitive receivers is based on software defined radio (SDR) technology that enables through software, dynamic reconfiguration of all protocols stacks including the physical layer. In other words, frequency band, air-interface protocol, and functionality can be upgraded with software download and update instead of a complete hardware replacement. SDR provides an efficient and secure solution to the problem of 
building multi-mode, multi-band, and multi-functional wireless communication devices [7]. A cognitive radio (CR) is an SDR that additionally senses its environment, tracks changes, and reacts upon its findings.

The main components of a cognitive radio transceiver are the radio front-end and the baseband processing unit. In the RF front-end, the received signal is amplified and mixed and is analog to digital converted [6]. The output of the digital front-end is then fed into the baseband processing engine. Each component must be able to be reconfigurable via a control bus. Note that a baseband processing engine can service multiple RF front-ends, each of which supports specific airinterface standards. The baseband processing unit has first to detect the presence of a signal by any wellknown techniques in the literature $[25,37], \ldots$ and then identify the systems corresponding to the detected signal. The identification of OFDM systems has been addressed in many papers, with different approaches. The reader can refer to [38-41] for example. Once the system has been identified, according to the protocol used by this system, the baseband processing unit will start and estimation of the relevant metrics using our proposed algorithms in Sections 2 or 3. When the metrics are estimated, an interaction needs to be performed with the higher layers to decide whether to trigger a vertical handover or not. A block diagram of the receiver is illustrated in Figure 5.

\section{Simulation and experimental results} 5.1 Metrics for CSMA/CA based networks

In this section, we present computer simulations results that show the algorithms performance.

\subsubsection{SNR estimation}

In this section, the performance of the proposed estimator is assessed on WiFi signals. WiFi signals are OFDM signals with 64 subcarriers and a guard interval of length equal to 16. The propagation channel $\{h(l)\}_{l}=0, \ldots$, $L-1$ has an exponential decay profile for its non-null component (i.e., $\mathbb{E}\left[|h(l)|^{2}\right]=G e^{-l / \mu}$ for $l=0, \ldots, L-1$ ), $G$ is chosen such that $\sum_{l=0}^{L} \mathbb{E}\left[\left|h_{k}(l)\right|^{2}\right]=1$. The channel is assumed to be time variant with a Doppler frequency equal to $10 \mathrm{~Hz}$ for WiFi signals and a root-mean-square delay spread of $25 \%$ of $D$.

The SNR is processed as described in Section 2.1. In Figure 6, we plot the normalized mean square error (NMSE) of the SNR estimation versus the true SNR for different $\mathcal{M}_{s}, \quad \mathrm{NMSE}=\mathbb{E}\left[\left(\hat{S} / \hat{\sigma}_{w}^{2}-S / \sigma_{w}^{2}\right)^{2} \frac{\sigma^{4}}{S^{2}}\right]$. Our method is compared with the approximate maximum likelihood (AML) estimator described in [14]. This estimator relies on an empirical threshold $\alpha$ that is used to determine the channel length which is required to estimate the SNR. The choice of this threshold, as described in [14], is subjective. If alpha is too small, the channel length will be overestimated, resulting in a poor efficiency of the estimator. If it is too large, signal samples are included in the noise variance estimator, leading to an underestimation of the SNR. $\alpha$ is here set to 0.05 ; this choice is empirical in our algorithm [13] and has been compared to the one in [14] for many values of $\alpha$ and always outperforms it. The reader can refer to [13] for more details on the impact of $\alpha$. Figure 6 highlights two limitations of the AML algorithm. First, as previously explained, this method depends on the subjective threshold $\alpha$, which has a strong impact on the








Figure 6 NMSE on the estimation of the SNR value.

performance. Then, as the signal power and noise variance estimations are not independent, the SNR estimation gets deteriorated at low and high SNR. Moreover, Figure 6 reveals that the algorithm presented in this paper globally outperforms the AML.

WiFi supports a large number of modulation and forward error correction coding schemes and allows to change it based on the channel conditions (adaptive modulation and coding (AMC)). The objective of AMC is to maximize the throughput in a time-varying channel. Since the adaptation algorithm typically calls for the use of the highest modulation and coding scheme that can be supported for the current SNR, it is possible to know the used data rate. In Figure 7, we plot the probability of estimating the SNR within the range of $\pm 1 \mathrm{~dB}$ of the true value. It clearly indicates that our SNR

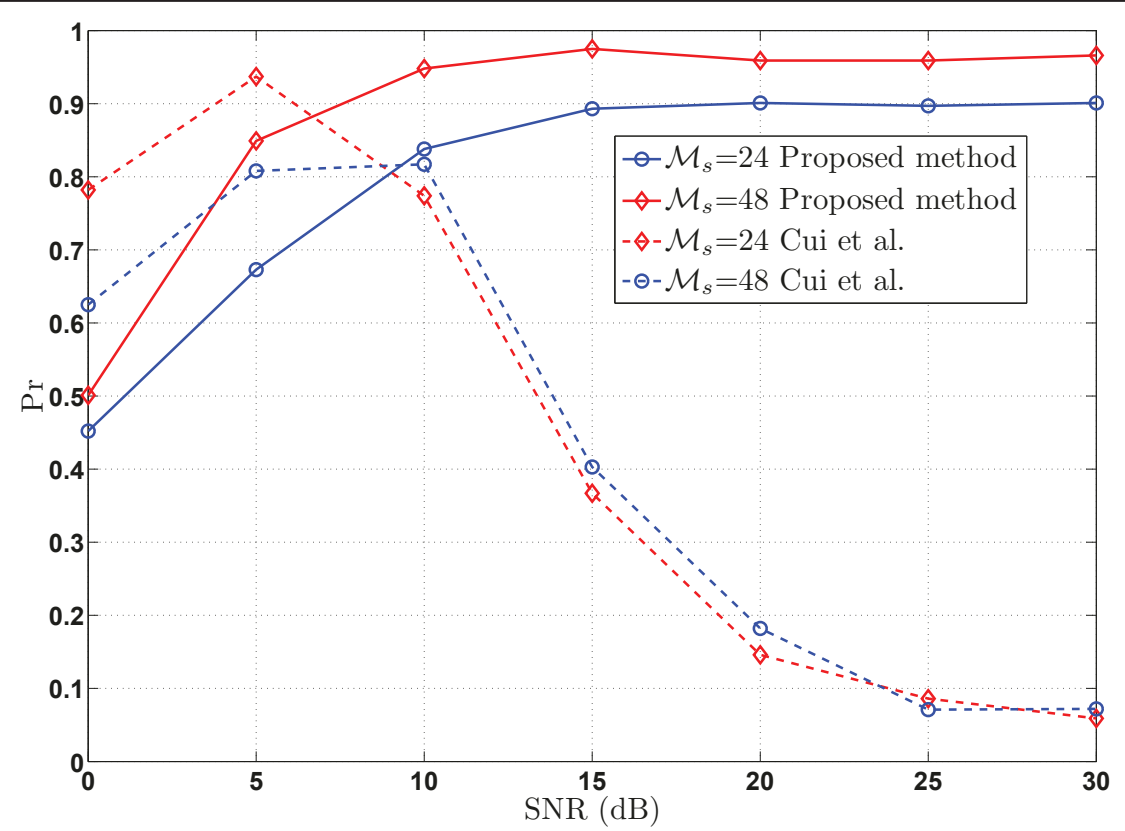

Figure 7 Probability of estimating the SNR within $\pm 1 \mathrm{db}$ of the true value. 


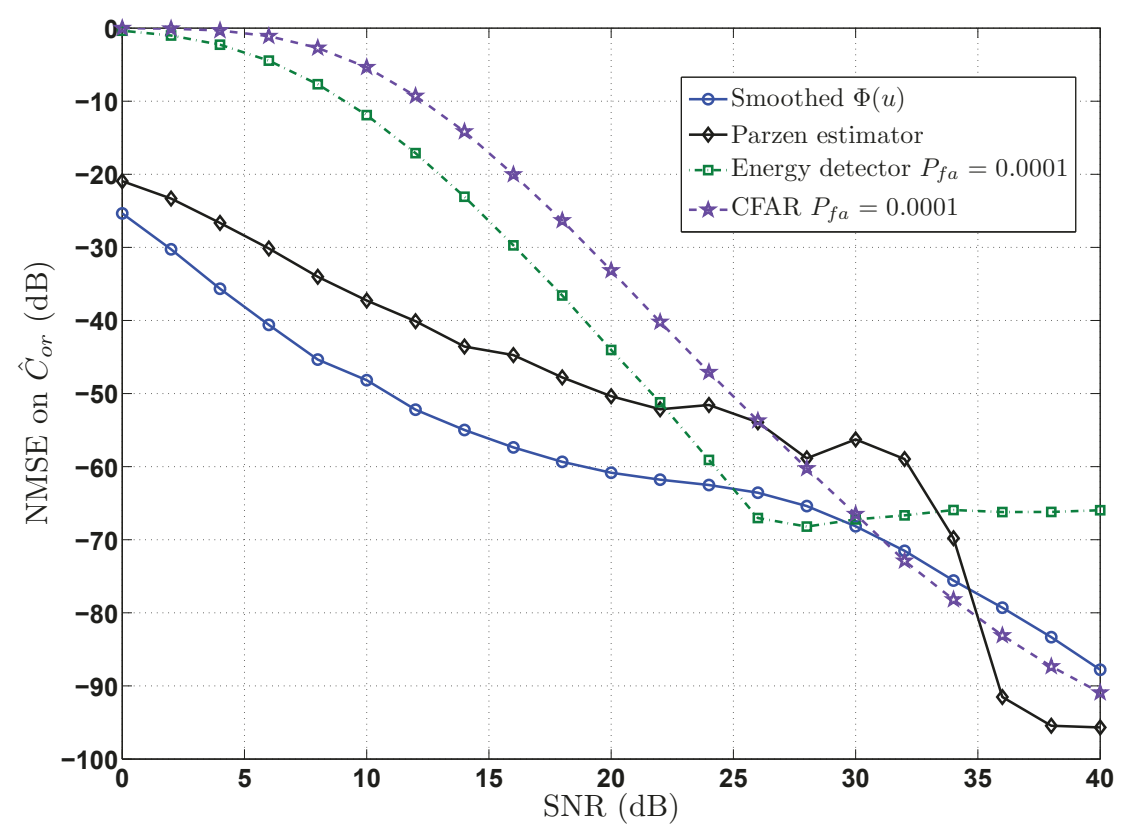

Figure 8 NMSE of the channel occupancy rate versus SNR.

estimator gives a reliable measure that can be used for vertical handoff decision. Note that this probability becomes greater than $80 \%$ for $\mathcal{M}_{s}=24$ and a SNR $\geq 0$ $\mathrm{dB}$ if the tolerated range is increased to $\pm 2 \mathrm{~dB}$.

\subsubsection{Channel occupancy rate}

In Figure 8, we show the NMSE (normalized mean square error) of the estimation of the channel occupancy rate versus the SNR. The results are averaged over 500 Monte Carlo runs, and the NMSE is here defined as $\mathbb{E}\left[\left(\widehat{C}_{o r, k}-C_{o r}\right)^{2} / C_{o r}^{2}\right]$, where $\widehat{C}_{o r, k}$ is the channel occupancy rate estimated at the $k$ th realization and $C_{o r}$ is the true channel occupancy rate. In this figure, we plot the performance of the estimator based on a smoothed $\Phi(u)$ criterion and a Parzen-based estimator. The Parzen estimator is also smoothed.

The proposed method is compared with the CFAR (constant false alarm rate) method with a probability of false alarm $P_{f a}=10^{-4}$ and with the energy detector proposed by Urkowitz [25], with a $P_{f a}=10^{-4}$. The cognitive terminal is supposed to have $N=2$ antennas. We can clearly see that the proposed approach outperforms the other methods.

Figure 9 shows the NMSE of the $C_{o r}$ estimated with a smoothed $\Phi(u)$ for different SNR versus the spectral occupancy rate. We can clearly see that the performance of the proposed method depends on the channel occupancy rate value. However, even for low $C_{o r}$, the method is very accurate $(-49 \mathrm{~dB})$.

As stated previously, the criterion has validation limits, and for a certain range of the noise variance, it behaves badly. To fix this problem, we proposed the Parzen estimator and stated that it does not depend on the noise variance. Figure 10 shows the NMSE of the three proposed methods versus the noise variance value, the SNR is fixed to $15 \mathrm{~dB}$, and the channel occupancy rate is equal to $64 \%$. For this SNR value, the criterion should be valid for: $2.16 \times 10^{-15}<\sigma_{w}^{2}<0.1171$. In the figure, the lower bound corresponds to $1 / \pi e^{1+\gamma}=2.16 \times 10^{-15}$ and the upper bound to $1 / \pi e=0.1171$. We can clearly see that only the Parzen estimator-based method is not affected by the noise variance value.

\subsubsection{Collision detection}

Figure 11a and 11b show the performance of the proposed method versus SNR. We clearly see that for both AIC and MDL, we get a good probability of detection for a SNR greater than $10 \mathrm{~dB}$, which is the usual operating range of the WiFi. Note that there is no motivation to trigger a vertical handover toward an access point that does not satisfy the signal strength condition. The simulations were done with an observation window of $40 \mu$ s length. We observe that AIC behaves better than MDL. The simulations were processed on frames whose starting, and ending points are supposed to be perfectly known.

\subsubsection{Experimental results}

The proposed blind algorithm for the estimation of the channel occupancy rate of a WiFi AP is evaluated using the RAMMUS RF platform developed in the Signal \& Communications department of TELECOM Bretagne. The aim of the experiments was not to highlight the precision of the algorithm since the true $C_{o r}$ is not available but to highlight the efficiency of the proposed 


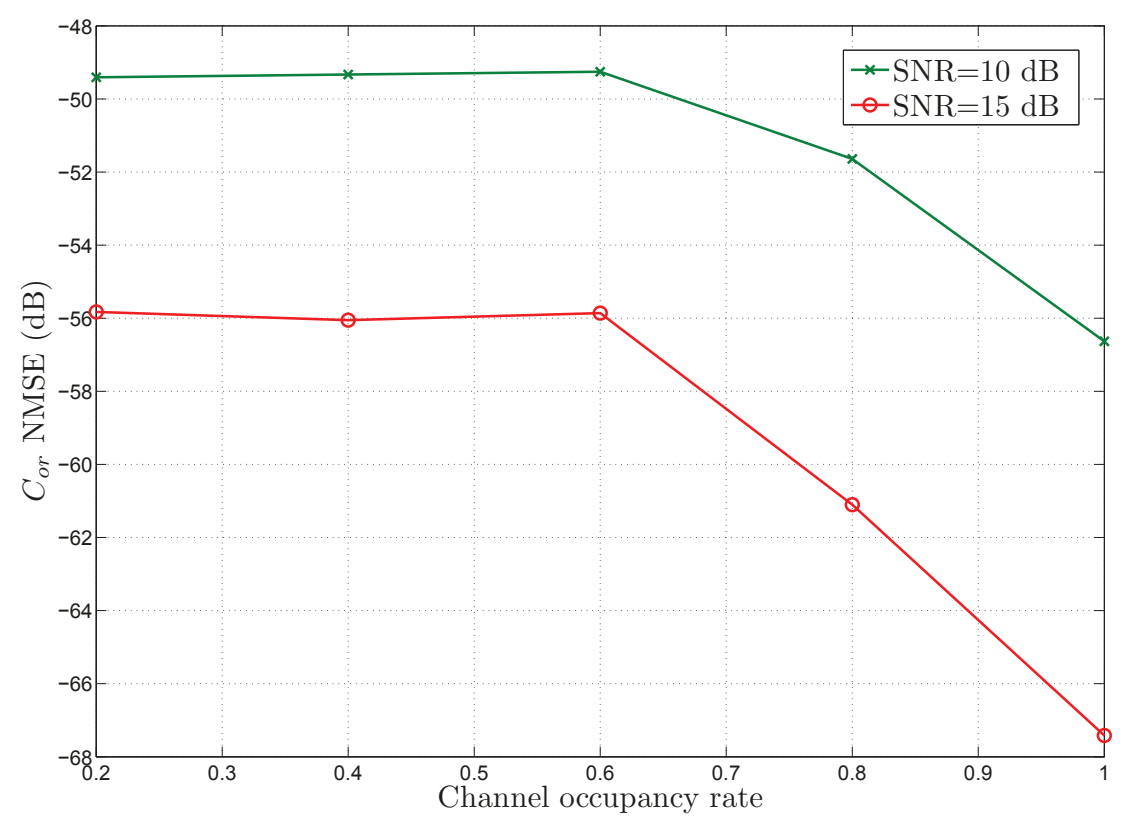

Figure 9 NMSE of the smoothed $\Phi(u)$ proposed method versus the channel occupancy rate.

metric in different scenarios. Experiments were investigated on the Channel $6(2.437 \mathrm{GHz})$ using the IEEE $802.11 \mathrm{~g}$ norm. We tested different schemes with different number of users for different maximum bit rate allocated to each user. The schemes are based on Client/ Server systems using the User Datagram Protocol (UDP) as presented in Figure 12. The physical layer signal is captured thanks to an USRP2 device (Universal Software
Radio Peripheral [42]). The sampling rate is set to 20 Mega-samples/s. The traffic rate is controlled thanks to J-Perf which is a software for UDP/TCP traffic generation, and the list of used equipments is illustrated in Table $1^{\mathrm{c}}$.

The observation window varies from 1 to $10 \mathrm{~ms}$, and the presented results were averaged over 500 non-correlated experiments. We test three scenarios varying the

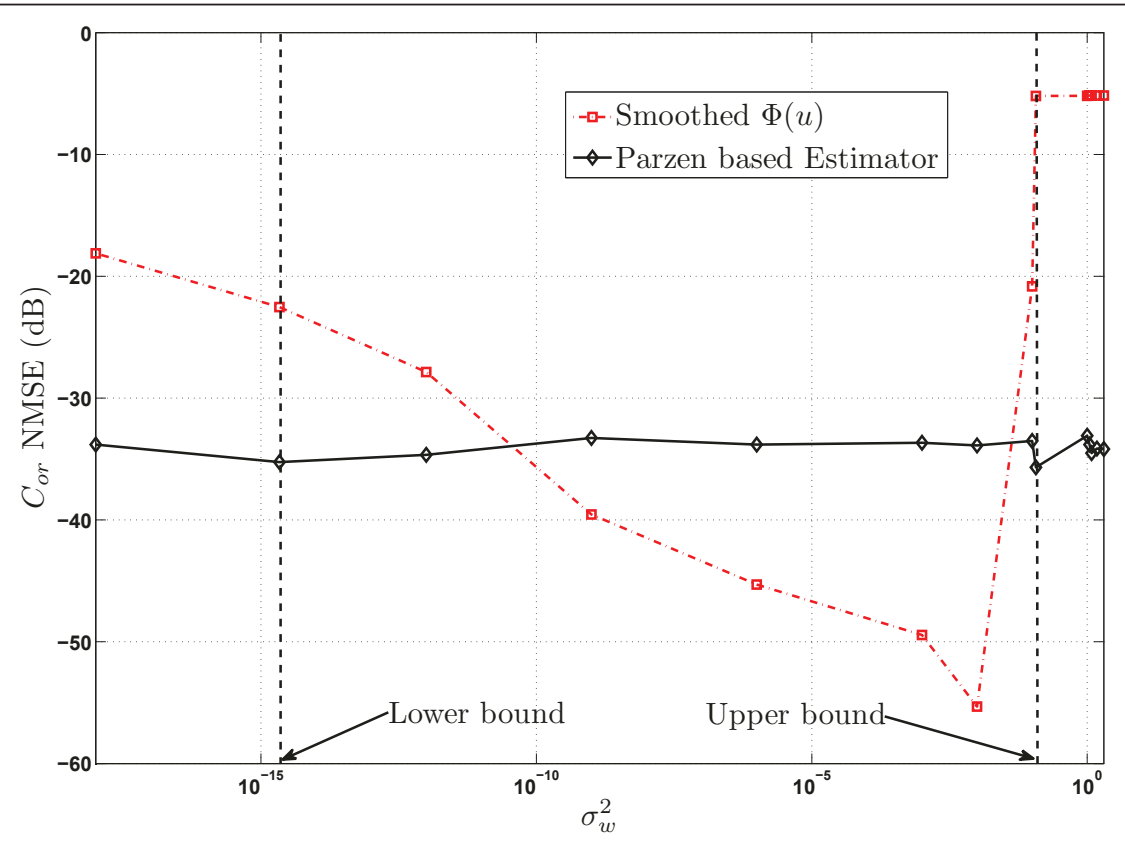

Figure 10 NMSE versus noise variance value, constant SNR. 


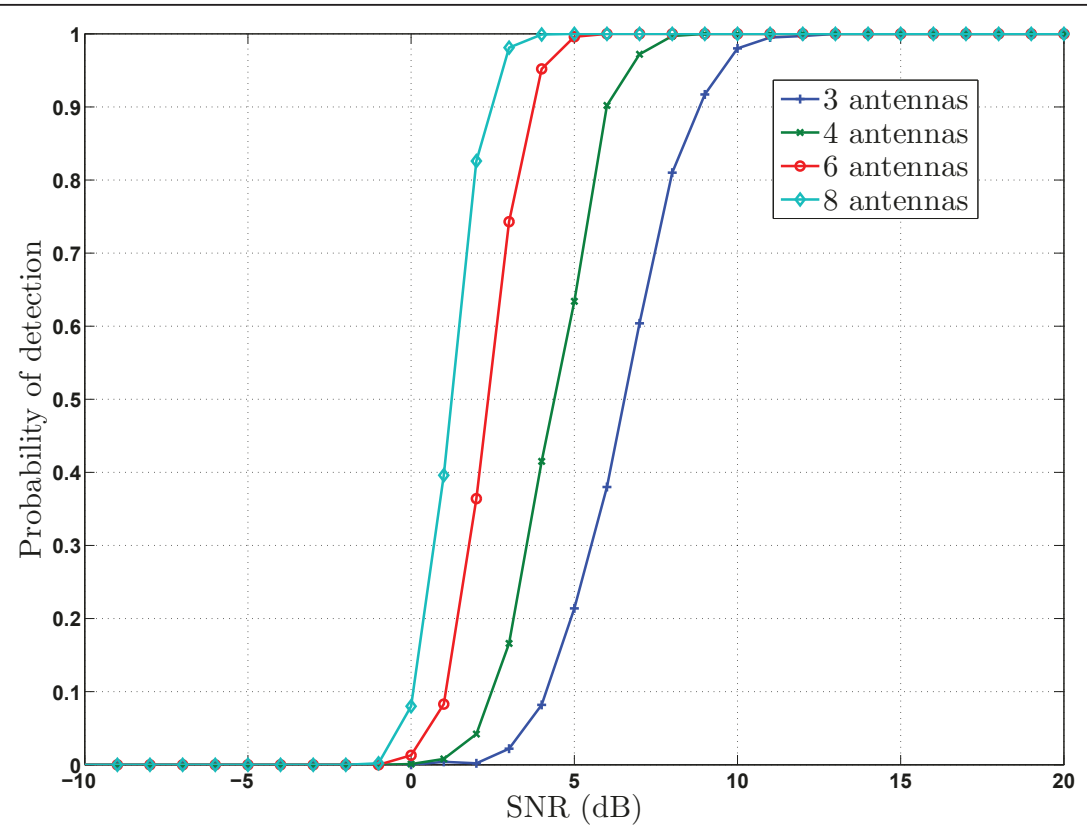

(a) Probabilty of detection with AIC versus SNR

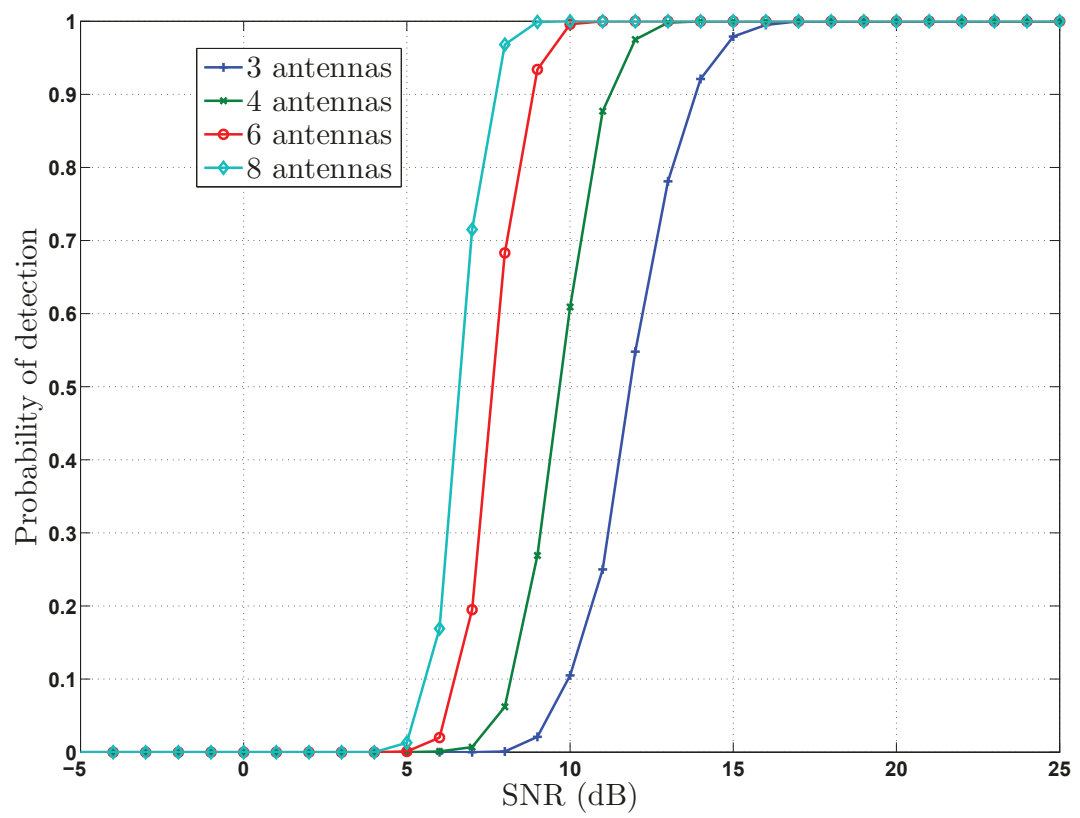

(b) Probabilty of detection with MDL versus SNR

Figure 11 Influence of the SNR on the probability of detection. (a) Probability of detection with AIC versus SNR; (b) Probability of detection with $M D L$ versus SNR.

number of $\mathrm{C} / \mathrm{S}$ systems from one to three. Each $\mathrm{C} / \mathrm{S}$ couple is exchanging data at a $1 \mathrm{Mbps}$ rate. The results are shown in Figure 13a. We clearly see that as the number of users increases, the channel occupancy rate increases too. In Figure 13b, we plot the variance of the estimated channel occupancy rate for one and three $\mathrm{C} / \mathrm{S}$ systems. It is obvious that for the shortest the observation window, the variance is the highest. Therefore, to 




Figure 12 Configuration of the used network for the experiments.

have the minimum variance, the observation window should be as long as possible, but for a seamless and a minimum latency handover, this window should be taken as short as possible. Concerning the selection of the observation window, it depends on the degree of accuracy desired by the user. The longer the observation window, the more accurate the estimator. However, in a vertical handover context, the user does not need to have an accurate estimation of the metric. He just needs to know approximately in which range is it and prefers certainly to decrease the scanning time, since using a long observation window increases the global scanning time, which is a crucial parameter that needs to be reduced to ensure a seamless and proactive handover.

In Figure 14, we show the influence of the data rate on the channel occupancy rate. For three Client/Server systems, we plot the channel occupancy rate for

Table 1 Configurations of the experiments

\begin{tabular}{lll}
\hline Equipment & Function & Quantity \\
\hline NETGERAR RangeMax & Router and access point, DHCP & 1 \\
WNR3500L & server & 6 \\
Dell Laptop Mobil Stations & Clients & 1 \\
Dell Laptop PHY Scanner & PHY Scanning and processing & 1 \\
USRP2 & Scanning PHY open hardware & 1 \\
& card & 3 \\
NETGEAR RangeMax & Wireless USB adapter & \\
WNDA3100 & & 3 \\
Intel(R) WiFi Link 5300 AGN & Integral wireless card & 6 \\
J-Perf Software & Traffic generator & \\
\hline
\end{tabular}

different data rate. Each system uses the same data rate. We observe that as the data rate increases, the channel occupancy rate increases in the same way. We also notice that the variance is lower for systems using higher data rates.

As explained previously, the aim of the algorithm is to trigger a vertical handoff toward the access point where the traffic is lower. According to the figures, we clearly see that the channel occupancy rate is lower in the configurations where a lower bit rate is required by users and increases as the required bit rate and number of users increases.

In Figure 15, we show the channel occupancy rate for different bit rates, the number of $\mathrm{C} / \mathrm{S}$ systems is set to one and the presented values are measured with a 4-ms observation window duration. We observe that for high data rates, the $C_{o r}$ reaches a certain value and does not change. This is due to the backoff intervals. More precisely in Figure 16 , we can see that the $C_{\text {or }}$ for three users is lower than the one for two users, this is due to the fact that for three users, the probability of collisions increases and then the used backoff are longer and the measured $C_{o r}$ decreases. In such a case, the $C_{o r}$ is not a good metric to trigger a $\mathrm{VHO}$, and the more appropriate metric is the one that we proposed for collision detection in Section 2.3.

\subsection{Metrics for OFDMA-based networks 5.2.1 OFDMA SNR estimation}

In this section, the performance of the proposed estimators is assessed on WiMAX signals. The configuration tested is a partial usage of subchannels configuration 


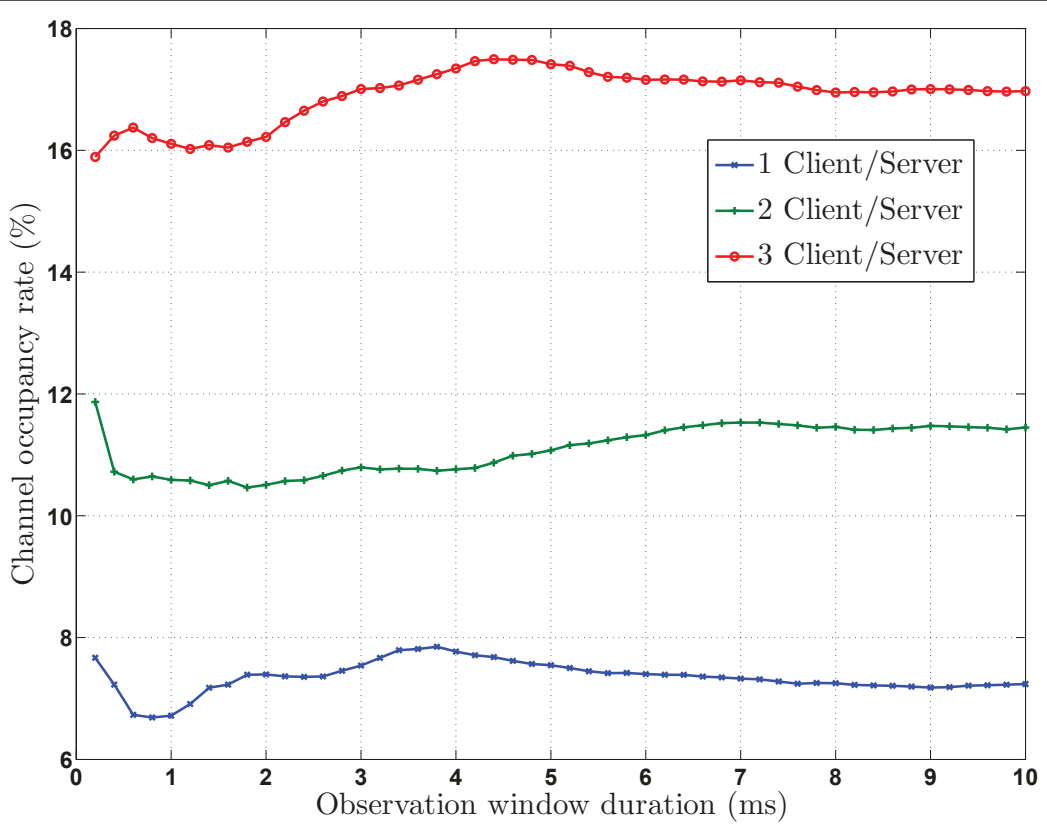

(a) $C_{\text {or }}$ versus the observation window duration



(b) $C_{\text {or }}$ versus the observation window duration with corresponding variance

Figure 13 Influence of the number of users on the channel occupancy rate. (a) $C_{o r}$ versus the observation window duration; (b) $C_{o r}$ versus the observation window duration with corresponding variance.

with 512 subcarriers (Section 8.4, Table 310.b, [35]), and $D$ is set to 64 . The propagation channel $\left\{h(l)_{l=0, \ldots, L}\right\}$ has an exponential decay profile for its non-null component (i.e., $\mathbb{E}\left[|h(l)|^{2}\right]=G e^{-l / \mu}$ for $l=0, \ldots, L$ ), and $G$ is chosen such that $\sum_{l=0}^{L} \mathbb{E}\left[\left|h_{k}(l)\right|^{2}\right]=1$. The channel is assumed to be time variant with a Doppler frequency equal to $100 \mathrm{~Hz}$ for WiMAX signals and a root-meansquare delay spread of $25 \%$ of $D$. 


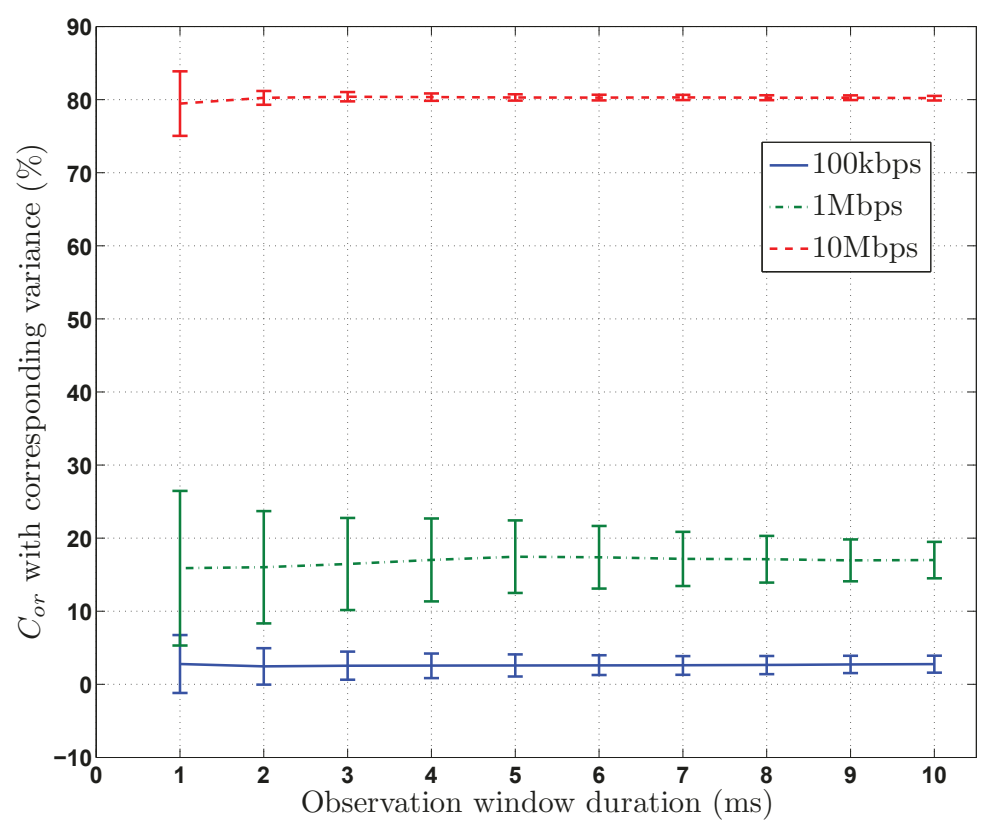

Figure $14 C_{\text {or }}$ versus the observation window duration with corresponding variance for different data rate

The SNR is processed as described in Section 3.1. In Figure 17, we plot the normalized mean square error (NMSE) of the SNR estimation versus the true SNR for different $\mathcal{M}_{s}$. Our method is compared with the approximate maximum likelihood (AML) estimator described in [14], while the threshold $\alpha$ is set to 0.05 . Once again, Figure 17 reveals that the algorithm presented in this paper globally outperforms the AML.
In Figure 18, we plot the probability of estimating the SNR within the range of $\pm 1 \mathrm{~dB}$ of the true value. It clearly indicates that our SNR estimator gives a reliable measure that can be used for vertical handoff decision. Note that this probability becomes greater than $97 \%$ for $\mathcal{M}_{s}=24$ and a SNR $\geq 0 \mathrm{~dB}$ if the tolerated range is increased to $\pm 1.5 \mathrm{~dB}$.

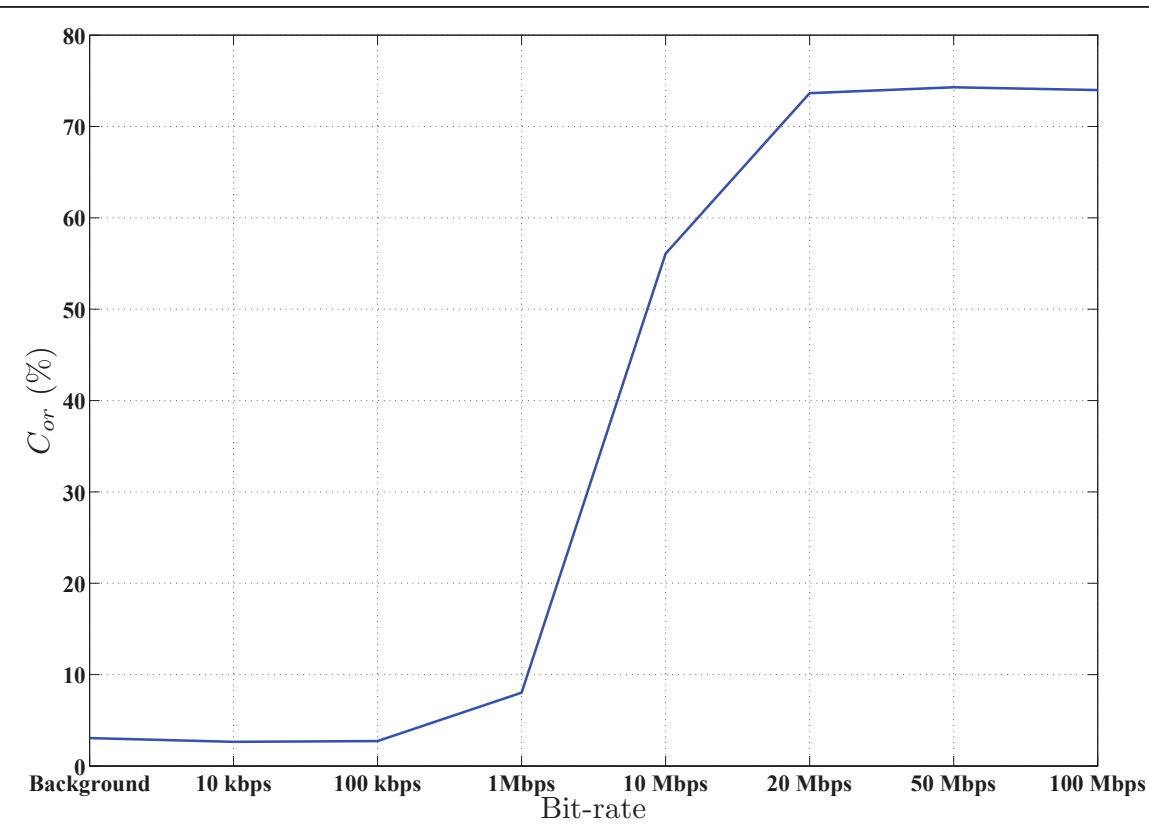

Figure $15 C_{o r}$ versus different bit rates. 


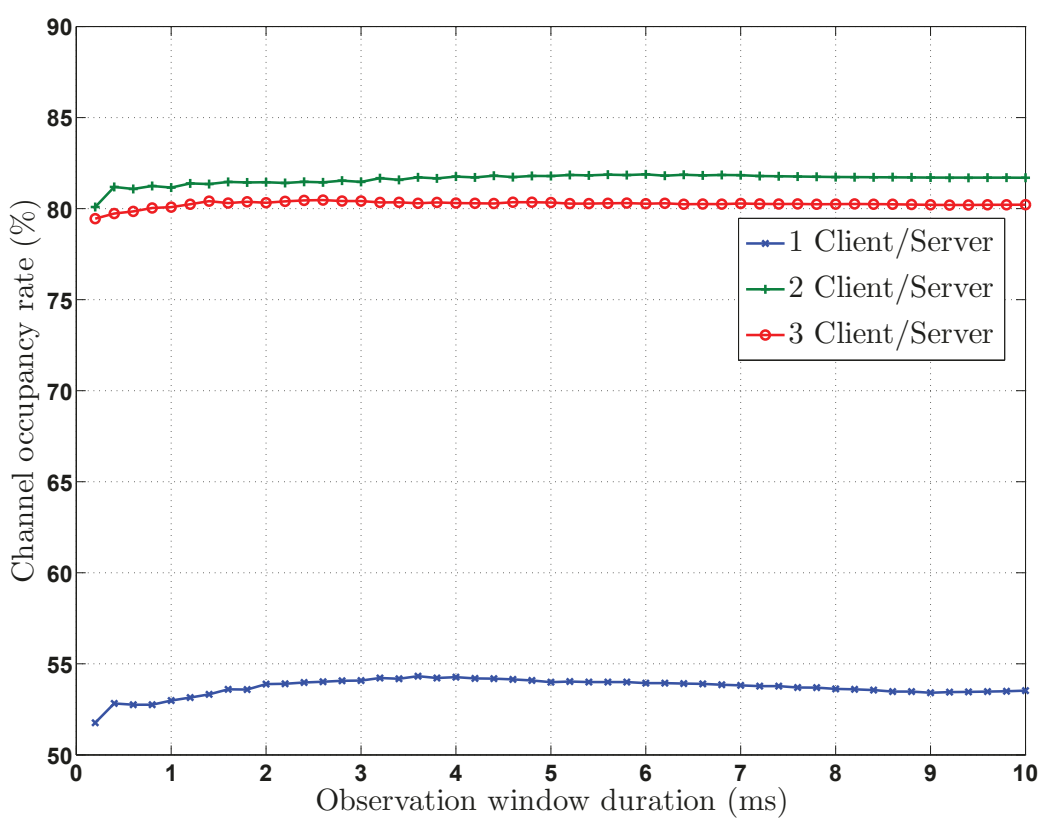

Figure $16 C_{\text {or }}$ versus the observation window duration, data rate $=10 \mathrm{Mbps}$

\subsubsection{OFDMA time-frequency activity rate estimation}

In this section, OFDMA signals with 512 subcarriers are considered. $D$ is set to $128, M=24$, and the time-frequency slots' allocation is supposed i.i.d. Each $a_{k, n}$ is randomly chosen within BPSK, QPSK, 16-QAM, and 64-QAM constellations according to a uniform law. These constellations are the main constellations used by the WiMAX adaptive modulation and coding (AMC) scheme [35].

The estimator performance is assessed in WMAN (wireless metropolitan area networks) environment where the channel is highly selective [43]. Figure 19 shows the NMSE (normalized mean square error) of the proposed estimators for different SNR versus the activity



Figure 17 NMSE on the estimation of the SNR value. 


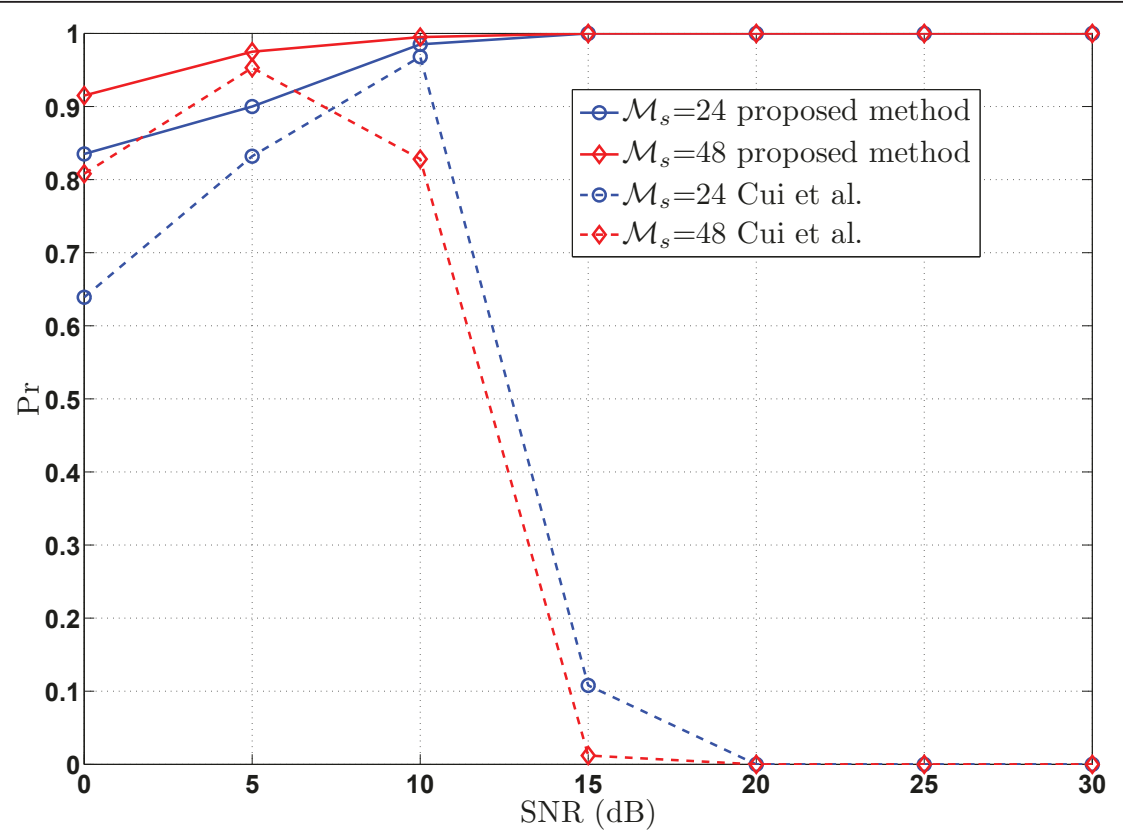

Figure 18 Probability of estimating the SNR within $\pm 1 \mathrm{db}$ of the true value.

rate. The propagation channel $\left\{h_{k}(l)\right\}_{l=0, \ldots, L}$ has an exponential decay profile for its non-null component (i. e., $\mathbb{E}\left[\left|h_{k}(l)\right|^{2}\right]=G e^{-l / \mu}$ for $\left.l=0, \ldots, L\right)$ with $L=D, \mu=32$ and $G$ is chosen such that $\sum_{l=0}^{L} \mathbb{E}\left[\left|h_{k}(l)\right|^{2}\right]=1$. The channel is assumed to be time variant with a Doppler frequency equal to $100 \mathrm{~Hz}$. Figure 19 compares the performance of the estimator in the two cases where $\sigma_{w}$ is first assumed to be perfectly known and when it is estimated thanks to the method presented in [13] and [34]. We observe that the estimator's performance deteriorates when $\sigma_{w}$ is estimated but still offers satisfying performance for the targeted application. A NMSE of $-15 \mathrm{~dB}$ can indeed be considered as sufficiently accurate to decide whether to trigger a handover or not.

In Figure 20, the performance of the proposed estimator is compared with that of the constant false alarm

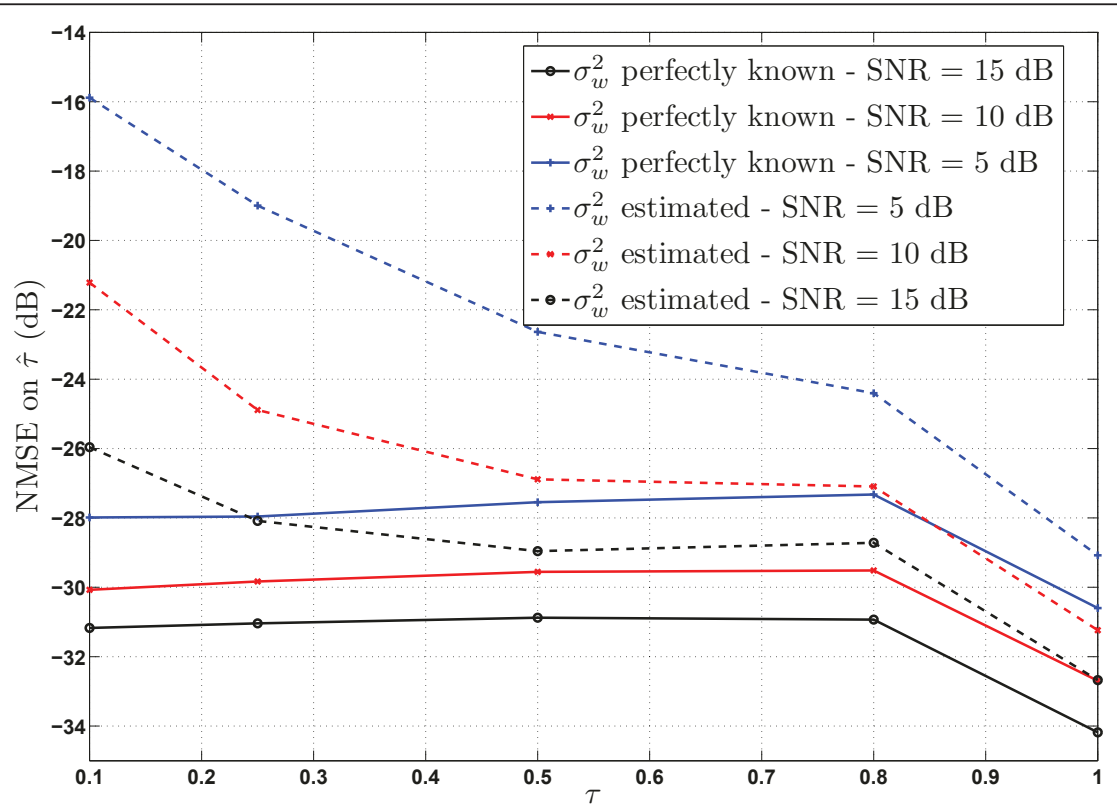

Figure 19 NMSE of the proposed method versus the activity rate, $\sigma_{w}$ known and estimated. 


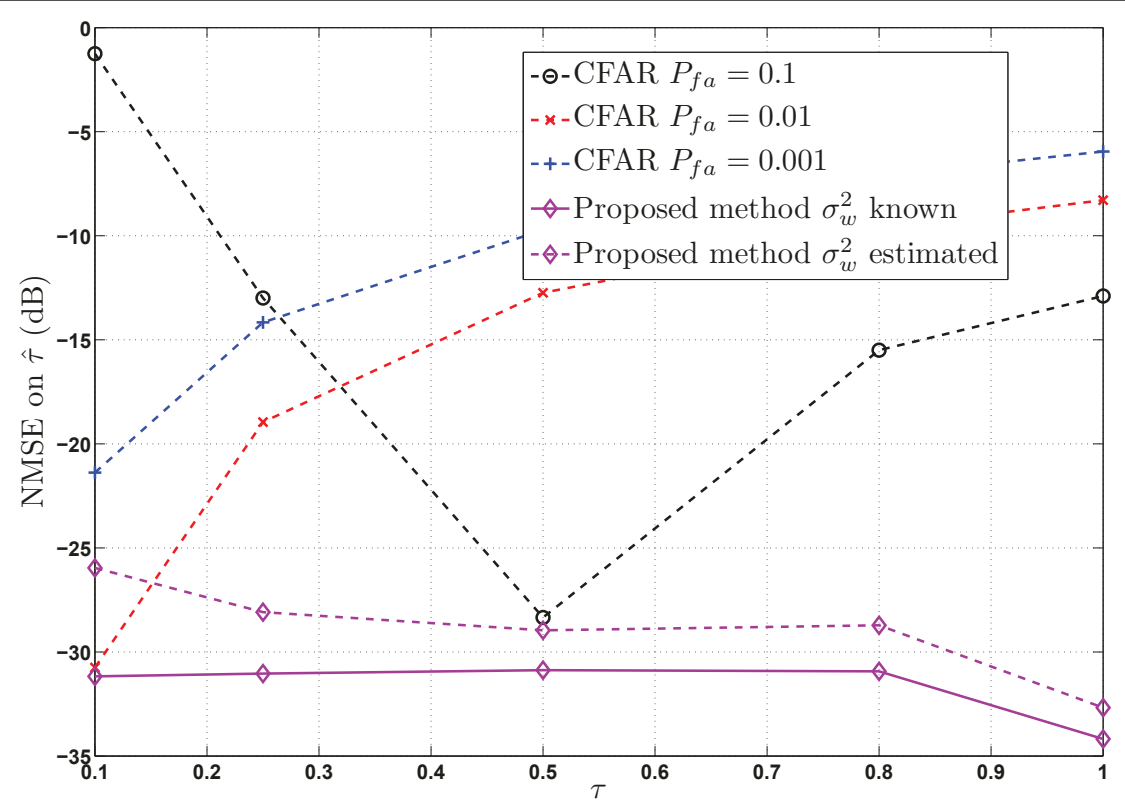

Figure 20 NMSE of the proposed method compared to the CFAR method.

rate (CFAR) technique [28]. The results show the problems induced by the choice of the threshold. We clearly observe that for a given $\mathrm{SNR}=10 \mathrm{~dB}$, the choice of the threshold greatly impacts the performance of the CFAR method. The proposed approach offers better and more stable results even when $\sigma_{w}$ is estimated.

Figure 21 compares the performances of the proposed algorithm in the case where the $a_{k, n}$ are not uniformly chosen from one of the possible constellation available in WiMAX. Indeed, we assessed simulations where the probability that a chosen symbol belongs to any constellation is $\mathrm{P}\left(a_{k, n} \in \mathrm{BPSK}\right)=\frac{1}{10}, \quad \mathrm{P}\left(a_{k, n} \in \mathrm{QPSK}\right)=\frac{1}{10}$, $\mathrm{P}\left(a_{k, n} \in 64-\mathrm{QAM}\right)=\frac{4}{10}, \quad \mathrm{P}\left(a_{k, n} \in 64-\mathrm{QAM}\right)=\frac{4}{10}$. We clearly note that the proposed algorithm is robust to a non-equirepartition of the constellation due to the AMC scheme.

Finally, Figure 22 compares the performance of the algorithm for various values of the observed number of



Figure 21 NMSE of the proposed method in the case of uniform and non-uniform repartition of the constellations 


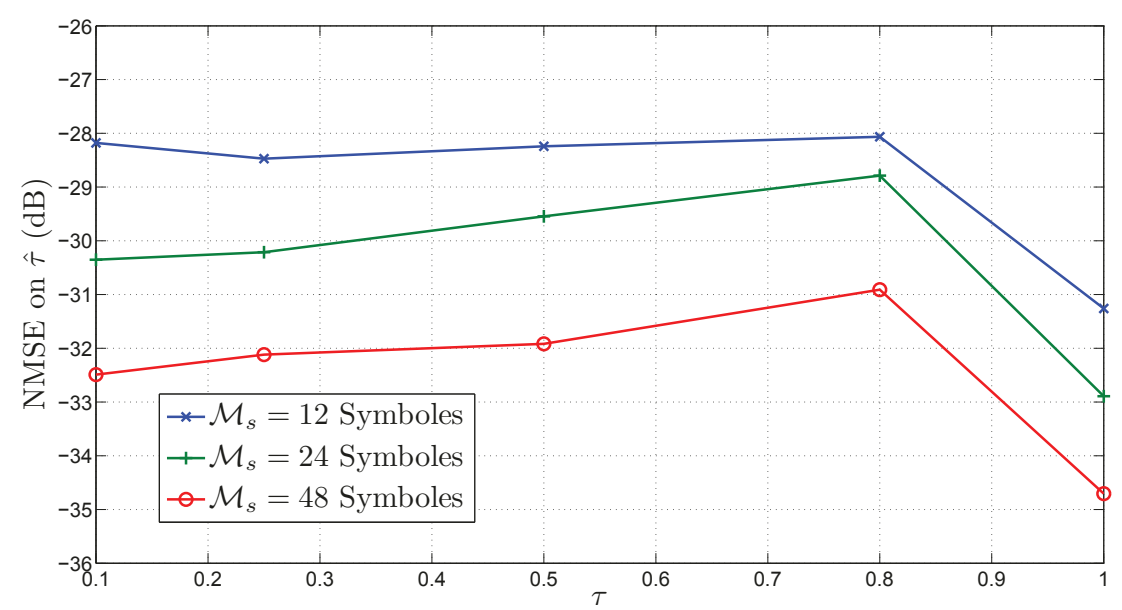

Figure 22 NMSE of the proposed method for various number of observed symbols, SNR $=10 \mathrm{~dB}$.

symbols $\mathcal{M}_{s}$, and the $\mathrm{SNR}$ is fixed to $10 \mathrm{~dB}$. As expected, the performance increases as the number of symbols increases. This can be justified intuitively, as more symbols are observed, as much information about the estimated parameters is available. It is obvious that it cannot be increased arbitrarily, since it determines the time lag before a decision is made, which again is a crucial parameter that needs to be reduced to ensure a seamless and proactive handover.

\section{Conclusion}

When the QoS offered to a mobile station does not satisfy the upper layer application, the latter needs to migrate between heterogeneous networks looking for better performance. As a previous step to the vertical handover, a sensing step of the QoS of the present networks is needed. Since these networks rely on different medium access mechanisms, methods to estimate the link quality have to be adapted to each of them.

New metrics for vertical handover toward OFDM systems have been proposed in this article. First, we proposed a method to get the SNR for OFDM-based systems. SNR is the most relevant indicator of the link quality but not always sufficient. Therefore, we focused on the CSMA/CA-based systems and propose to estimate two metrics: The first one is related to the channel occupancy rate and the second one to the collision rate. These two metrics inform us on the MAC-layer QoS of the network, such as available bandwidth and access delay, which are relevant to trigger a vertical handover if combined with the SNR. Computer simulation and experimentation are run on WiFi signal (most famous CSMA/CA-based system). Good performances are obtained for the WiFi SNR operating range.

Then, a new blind estimation method of OFDMA time-frequency activity rate has been presented. The method is computationally cheap and exhibits accurate estimation. This approach does not rely on a choice of a threshold and shows good performance compared with the classical CFAR approach even when the noise variance $\sigma_{w}$ is estimated.

All the proposed algorithms are blind and rely only on a physical layer sensing, which makes them low computational and avoid time and power waist to get connected $^{\mathrm{d}}$.

\section{End notes}

${ }^{a}$ Note that the intercell interference is neglected here. ${ }^{\mathrm{b}}$ SIMO model is considered here, where multiple antennas at reception are required to estimate the noise variance with no frame synchronization. The proposed technique is also valid in the SISO case, but the noise variance must be known. ${ }^{\mathrm{c}}$ Thanks to $\mathrm{S}$. HADIN, the research engineer who realized the experiments. ${ }^{d}$ The authors declare that they have no competing interests.

Received: 12 January 2011 Accepted: 12 September 2011 Published: 12 September 2011

\section{References}

1. J McNair, F Zhu, Vertical handoffs in fourth-generation multinetwork environement. IEEE Trans Wirel Commun. 11, 8-15 (2004)

2. W-T Chen, J-C Liu, H-K Huang, An adaptive scheme for vertical handoff in wireless overlay networks, in Parallel and Distributed Systems, International Conference on. 0, 541 (2004)

3. U Pineda-Rico, E Stevens-Navarro, J Acosta-Elias, Vertical handover in beyond third generation (B3G) wireless networks. Int J Futur Gener Commun Netw. 1, 51-58 (2008)

4. Simon Haykin, Cognitive radio: brain-empowered wireless communications. IEEE J Sel Areas Commun. 23(2), 201-220 (2005)

5. J Mitola, GQ Maguire, Cognitive radio: making software radios more personal. IEEE Pers Commun. 6, 13-18 (1999). doi:10.1109/98.788210

6. I-F Akyildiz, W-Y Lee, M-C Vuran, S Mohanty, Next generation/dynamic spectrum access/cognitive radio wireless networks: a survey. Comput Netw Elsevier. 50(13), 2127-2159 (2006). doi:10.1016/j.comnet.2006.05.001 
7. F-K Jondral, Software-defined radio basics and evolution to cognitive radio. EURASIP J Wirel Commun Netw. 3, 275-283 (2005)

8. E Adamopoulou, K Demestichas, M Theologou, Enhanced estimation of configuration capabilities in cognitive radio. IEEE Commun Mag. 46(4), 56-63 (2008)

9. F Zhu, J McNair, Optimizations for vertical handoff decision algorithms, in Wireless Communications and Networking Conference, 2004. WCNC. 2004 IEEE. 2, 867-872 (March 2004)

10. W Zhang, Handover decision using fuzzy MADM in heterogeneous networks, in Wireless Communications and Networking Conference, 2004. WCNC. 2004 IEEE. 2, 653-658 (2004)

11. Q Song, A Jamalipour, A network selection mechanism for next generation networks, in Communications, 2005. ICC 2005. 2005 IEEE International Conference on. 2, 1418-1422 (May 2005)

12. Z Dai, R Fracchia, J Gosteau, P Pellati, G Vivier, Vertical handover criteria and algorithm in IEEE 802.11 and 802.16 hybrid networks, in IEEE International Conference on Communications, pp. 2480-2484 (2008)

13. F-X Socheleau, A Aissa-El-Bey, S Houcke, Non data-aided SNR estimation of OFDM signals. IEEE Commun Lett. 12(11), 813-815 (2008)

14. T Cui, C Tellambura, Power delay profile and noise variance estimation for OFDM. IEEE Commun Lett. 10(1), 25-27 (2006). doi:10.1109/ LCOMM.2006.1576558

15. P Jallon, An algorithm for detection of DVB-T signals based on their second-order statistics. EURASIP J Wirel Commun Netw. 2008, 28:1-28:9 (2008)

16. J van de Beek, M Sandell, P Borjesson, ML estimation of time and frequency offset in OFDM systems. IEEE Trans Acoust Speech Signal Process. 45, 1800-1805 (1997)

17. $\mathrm{S} M a, X$ Pan, $G$ Yang, $T$ Ng, Blind symbol synchronization based on cyclic prefix for OFDM systems. Vehicular Technol IEEE Trans. 58(4), 1746-1751 (2009)

18. C Guo, Z Guo, Q Zhang, W Zhu, A seamless and proactive end-to-end mobility solution for roaming across heterogeneous wireless networks. IEEE J Sel Areas Commun. 22, 834-848 (2004). doi:10.1109/JSAC.2004.826921

19. Q Zhang, C Guo, Z Guo, W Zhu, Efficient mobility management for vertical handoff between WWAN and WLAN. Commun Mag IEEE. 41, 102-108 (2003)

20. M-R Oularbi, A Aissa-El-Bey, S Houcke, Physical Layer IEEE 802.11 Channel Occupancy Rate Estimation, in ISIVC 2010: International Symposium on ImagesNideo Communications over Fixed and Mobile Networks, October 2010 (2010)

21. $X X u, Y$ Jing, $X Y$ Y , Subspace-based noise variance and SNR estimation for OFDM systems, in Wireless Communications and Networking Conference, 2005 IEEE. 1, 24-26 (2005)

22. SM Kay, Fundamentals of statistical signal processing, Volume II: Detection Theory (Prentice Hall, Englewood Cliffs, 1998)

23. HL Van Tress, Detection, Estimation, and Modulation Theory, vol. I-III (Wiley, London, 1968-1971)

24. HL Van Tress, Elements of Signal Detection and Estimation (Prentice Hall, Englewood Cliffs, 1995)

25. H Urkowitz, Energy detection of unknown deterministic signals, in Proceeding of the IEEE. 55(4), 523-531 (1967)

26. DW Scott, Wiley series in probability and mathematical statistics: applied probability and statistic section, Multivariate Density Estimation: Theory, Practice, and Visualization (Wiley, London, 1992)

27. BW Silverman, Density Estimation for Statistics and Data Analysis (Chapman \& Hall/CRC Monographs on Statistics \& Applied Probability, London, 1992)

28. L Scharf, Statistical Signal Processing: Detection, Estimation, and Time Series Analysis (Addison Wesley, Reading)

29. J-H Yun, S-W Seo, Collision Detection based on RE Energy Duration in IEEE 802.11 Wireless LAN. Communication System Software and Middleware, 2006. Comsware 2006. First International Conference on. 0 (2006)

30. J Yun, S Seo, Novel collision detection scheme and its applications for IEEE 802.11 wireless LANs. Comput Commun Elsevier. 30(6), 1350-1366 (2007)

31. M Wax, T Kailath, Detection of signals by information theoretic criteria. IEEE Trans Acoust Speech Signal Process ASSP-33, 387-392 (1985)

32. M-R Oularbi, F-X Socheleau, A Aissa-El-Bey, S Houcke, Blind estimation of the time-frequency activity rate of OFDMA signals. in ICUMT 2010: International Conference on Ultra Modern Telecommunications, (October 2010)
33. B Park, E Ko, H Cheon, C Kang, D Hong, A blind OFDM synchronization algorithm based on cyclic correlation. IEEE Globecom Conf. 5, 3116-3119 (2001)

34. F-X Socheleau, D Pastor, A Aissa-El-Bey, S Houcke, Blind noise variance estimation for OFDMA signals. ICASSP 2581-2584 (2009)

35. IEEE Std 802.16, Part 16: air interface for broadband wireless access systems, Amendment 2: Physical and Medium Access Control layers for Combined Fixed and Mobile Operation in License Bands and Corrigendum 1 (2005)

36. ET Jaynes, in Probability Theory: The Logic of Science (Addison Wesley, NY, 2000)

37. D cabric, SM Mishra, RW Brodersen, Implementation issues in spectrum sensing for cognitive radios, in Proc. 38th Asimolar Conf. Sig., Sys. and Comp, pp. 772-776 (2004)

38. F-X Socheleau, S Houcke, P Ciblat, A Aissa-El-Bey, Cognitive OFDM system detection using pilot tones second and third-order cyclostationarity. Elsevier Signal Process. 91(2), 252-268 (2010)

39. H Li, Y Bar-Ness, A Abdi, O-S Somekh, W Su, OFDM modulation classification and parameters extraction, in 1st International Conference on Cognitive Radio Oriented Wireless Networks and Communications, pp. 1-6 (2006)

40. PD Sutton, KE Nolan, LE Doyle, Cyclostationary signatures in practical cognitive radio applications. IEEE J Sel Areas Commun. 26(1), 13-24 (2008)

41. A Bouzegzi, P Ciblat, P Jallon, New algorithms for blind recognition of OFDM based systems. Elsevier Signal Process. 90(3), 900-913 (2010)

42. USRP2, Ettus Research LLC website, http://www.ettus.com/ consulted April (2010)

43. V Erceg, et al, Channel Models for Fixed Wireless Applications, IEEE 802.16 Broadband Wireless Access Working Group (2001)

doi:10.1186/1687-1499-2011-93

Cite this article as: Oularbi et al:: Physical layer metrics for vertical handover toward OFDM-based networks. EURASIP Journal on Wireless Communications and Networking 2011 2011:93.

\section{Submit your manuscript to a SpringerOpen ${ }^{\circ}$ journal and benefit from:}

- Convenient online submission

- Rigorous peer review

- Immediate publication on acceptance

- Open access: articles freely available online

- High visibility within the field

- Retaining the copyright to your article

Submit your next manuscript at $\gg$ springeropen.com 